\title{
A new class of unconditionally stable numerical methods based on Adams Moulton's methods with application to radiative Darcy-Forchheimer flow
}

\author{
Yasir Nawaz ${ }^{1}$ and Muhammad Arif ${ }^{1}$ \\ ${ }^{1}$ Air University
}

April 28, 2020

\begin{abstract}
Present contribution has consisted of a construction of a class of new shooting methods and application to Darcy Forchheimer fluid flow over the linearly stretching sheet under the influence of thermal radiations. Governing equations of the flow phenomena are presented in the form of partial differential equations, and these governing partial differential equations are reduced into ordinary differential equations. The final ordinary differential equations are resolved by the present given shooting method based on the class of Adam Moulton's techniques with the Gauss-Siedel iterative method. Since the system of the first-order differential is discretized by the Adam Moulton method with the Gauss-Siedel iterative method containing some unknown initial conditions, a shooting approach is employed for finding unknown initial conditions. Also, it is proved that the Adam Moulton methods using Gauss-Seidel iterative method are unconditionally stable for any system of differential equations. In additions to this, finite element simulations containing velocity profile, streamlines, temperature profile and temperature contours are deliberated with the help of graphs, and tables. Thus, the main aim of the current paper is to calculate the unique results for a coupling approach based on Adams Moulton's method for the boundary layer flow problem with the effect of heat transfer.
\end{abstract} A new class of unconditionally stable Adams Moulton's numerical methods with application
to radiative Darcy-Forchheimer flow

Yasir Nawaz, Muhammad Shoaib Arif

Stochastic Analysis \& Optimization Research Group, Department of Mathematics, Air University, PAF Complex E-9, Islamabad, 44000, Pakistan.

Corresponding Email: shoaib.arif@mail.au.edu.pk

Abstract: Present contribution has consisted of a construction of a class of new shooting methods and application to Darcy Forchheimer fluid flow over the linearly stretching sheet under the influence of thermal radiations. Governing equations of the flow phenomena are presented in the form of partial differential equations, and these governing partial differential equations are reduced into ordinary differential equations. The final ordinary differential equations are resolved by the present given shooting method based on the class of Adam Moulton's techniques with the Gauss-Siedel iterative method. Since the system of the first-order differential is discretized by the Adam Moulton method with the Gauss-Siedel iterative method containing some unknown initial conditions, a shooting approach is employed for finding unknown initial conditions. Also, it is proved that the Adam Moulton methods using Gauss-Seidel iterative method are unconditionally stable for any system of differential equations. In additions to this, finite element simulations containing velocity profile, streamlines, temperature profile and temperature contours are deliberated with the help of graphs, and tables. Thus, the main aim of the current paper is to calculate the unique results for a coupling 
approach based on Adams Moulton's method for the boundary layer flow problem with the effect of heat transfer.

Keywords: Adams Moulton's methods; Gauss-Seidel method; Darcy-Forchheimer model; Radiations; Stability

\section{Introduction}

Today, development in technology has made integrated circuits in to tiny and support the research in nanomaterials. The in-depth study of stretchable flows gives a significant knowledge to the physics of boundary layer examination. These stretchable flows are systematically solved by reducing the boundary value problems into the self-comparable structure and then solved for numerical or series solution through various techniques. The trouble is that every stretching and shrinking problem consists of various governing equations, distinct transformations and a different system of solutions that are specific for each case. Scientists have formed the methods to resolve and model the flow produced by stretching and shrinking of plates [1-10]. Goldstein [11] studied various apparent structures in boundary layers which shrinking flow exhibit. Miklavcic and Wang [12] studied the flow of viscous material subjected to a shrinking sheet. The thermal manufacturing process involved heat energy conversion and its generation inside of the physical structure and are developed through heat transportation. Fourier [13] studied the utilisation of heat transportation in mechanical, biomedical and polymer industries. Wang et al. [14] have inspected the degree of thermal transportation of Nano liquids in the existence of carbon nanotubes.

Finding of fullerenes in 1985 and carbon nanotubules aroused the attention of scientific society towards their vast usage Kroto et al. [17]. Carbon has different allotropic forms and can adapt to various hybridisation states; in this term, CNTB is a single-dimensional structure that has extensive application in nanoelectronics Iijima [18]. These are made up of graphene (a two-dimensional structure that has been widely calculated by Ciraci et al. [19] and Novoselov et al. [20]) rolled into the cylinder and showed distinctive properties due to curvature Casari et al. [21]. Investigation in the field of nanotechnology is the principal aim of investigators, nowadays, which was first proposed by Choi in 1995. Nano-fluid, a crucial part of nanotechnology is capable of heat transfer and have applications in Textiles, electromagnetic, chemical, mechanical, optical and medicinal. Nadeem and Lee [24] have proposed the theory of nanofluids aver stretching sheet. Remarkable properties of nano liquids enable their frequent usage in nanotechnology. Diverse applications and vast and versatile approaches of these nanofluids can be studied in Sheikholeslami publications [25-30].

The fluid of nanomaterials is known as micropolar fluid, belong to the class of polar fluids. Eringen [30, 31] has presented the concept of micropolar fluids with their applications. Lukaszewicz [32] has shown the theory and usage of micropolar liquids. The micropolar liquid with the effects of the magnetic field was studied by Mohammeadein [33]. The inactivity (stagnation) point of flow in micropolar fluids was studied by Ishak et al. [34]. The effects of Joule heating on Magnetohydrodynamics micropolar and the effects of joule heating and thermal radiation on Magnetohydrodynamics micropolar liquid was studied by Hakaiem et al. [35] and Ramzan et al. [36] respectively; however, Yacob et al. [37] has presented the study of melting heat spread stagnation point flow over a stretching plate. Haque studied the Magnetohydrodynamics free mass movement and convection flow behaviour of micropolar liquid [38].

The system of the heat exchanger and system of geothermal energy are among those environmental and industrial systems that contain the convection flow with the existence of permeable medium. The standard law of Darcy is operative under little velocity and variable permeability. Forchheimer [42] has coincided a term square velocity to the Darcian's velocity by using inertia and boundary features; however, Muskat [43] has introduced "Forchheimer term", and for large Reynolds number, this term is permanently operative. The fluid movement and heat transfer study with rotating situation reveals its significant role in the petrol and chemical industries as well as in the study of earth, oceans and space technology. Greenspan and Howard [47] have found out the behaviour of rotating flow for viscous fluid which is filled in the asymmetric closed container.

The fluid movement through a porous medium has variable applications in thermal isolation, oil preparation 
and chemical reactors. [48-55] indicates energy and mass movement in various fluid models. Mostly the fluid movement via porous media is more promising with respect of groundwater, water flow in a reservoir, oil resources and several others.

The Darcy model is elaborated with increased flow rate and extended to non-Darcy with integrated properties of inertia and boundary layer features; however, Forchheimer [57] proposed revised version of this model named as Darcy-Forchheimer by announcing additional terms in momentum expression. Saddeek [58] coupled Darcy-Forchheimer flow with Brownian and thermophoresis diffusion over a permeable overextended surface. Hayat et al. [60] have calculated the flow as mentioned earlier with Cattaneo Christory heat changes; however, some new insight in a too in-depth investigation about Darcy-Forchheimer and overextended flows are in [61-66].

The present contribution is dealt with the coupling of three numerical methods and applied the coupling approach for solving ordinary differential equation obtained by using similarity transformation on the governing equation of the flow problem. Adams Moulton's methods are implicit, but the present way of applying Adams Moulton's methods can be considered as explicit because the techniques are coupled with the Gauss-Seidel method. Since the initial condition for every first-order is not given, so a shooting approach is considered, which is based upon the Matlab solver 'fsolve'.

\section{Problem Formulation}

Consider the Laminar, incompressible and unsteady fluid flow over the flat extending sheet. Let the rate of the stretching plate is $V=a x$. Let $x$-axis is considered parallel and coincides to the plate and $y$ - axis is perpendicular to the $x$ - axis or stretching plate. The fluid is considered with thermal radiations. Under these conditions with the use of the characteristic of Darcy-Forchheimer model with the consideration of heat transfer. The Governing equations are expressed as

$\frac{\partial u}{\partial x}+\frac{\partial v}{\partial y}=0(1)$

$u \frac{\partial u}{\partial x}+v \frac{\partial u}{\partial y}=\nu \frac{\partial^{2} u}{\partial y^{2}}-\frac{\nu}{K} u-F u^{2}(2)$

$u \frac{\partial T}{\partial x}+v \frac{\partial T}{\partial y}=\frac{k}{\rho c_{p}} \frac{\partial^{2} T}{\partial y^{2}}-\frac{1}{\rho c_{p}} \frac{\partial q_{r}}{\partial y}(3)$

Where $u$ be the horizontal and $v$ be the vertical components of velocity. Let $T$ be the temperature of the fluid, $\nu$ be the kinematic viscosity, $F=\frac{c_{b}}{x K^{1 / 2}}$ be the non-uniform inertia coefficient of the porous medium, $c_{b}$ be the drag coefficient, $K$ be the permeability of porous space, $\rho$ be the density, $c_{p}$ be the specified heat capacity, $k$ be the thermal conductivity and $q_{r}$ be the Roseland heat flux. The flow is considered with the following boundary conditions,

$$
\begin{gathered}
u=V, v=0, T=T_{w} \text { when } y=0 \\
u \rightarrow 0, T \rightarrow T_{\infty} \quad \text { when } y \rightarrow \infty
\end{gathered}
$$

Where $V=a x$ be the stretching velocity of the sheet, $T_{w}$ be the constant temperature of the wall and $T_{\infty}$ be a constant ambient temperature of the fluid. Using linearization of $T^{4}$ by $4 T T_{\infty}^{3}-3 T_{\infty}^{4}$, the Roseland heat flux can be reduced to

$q_{r}=\frac{-16 \sigma^{*} T_{\infty}^{3}}{3 k^{*}} \frac{\partial T}{\partial y}(5)$

Where $\sigma^{*}$ be a Stefan-Boltzman constant and $k^{*}$ be the mean absorption coefficient. The following transformations are used to reduce the set of partial differential equations (1) - (3) with boundary conditions $(4)$

$u=a x f^{\prime}(\eta), v=-(\alpha \nu)^{\frac{1}{2}} f(\eta), \eta=\left(\frac{a}{\nu}\right)^{\frac{1}{2}} y, \theta(\eta)=\frac{T-T_{\infty}}{T_{w}-T_{\infty}}$.

The reduce partial differential equations are expressed by 
$f^{\prime \prime \prime}(\eta)=\lambda f^{\prime}(\eta)+\left(1+F_{r}\right) f^{\prime}(\eta)^{2}-f(\eta) f^{\prime \prime}(\eta)(6)$

$\theta^{\prime \prime}(\eta)\left(1+\frac{4}{3} R_{d}\right)=-\operatorname{Pr} f(\eta) \theta^{\prime}(\eta)(7)$

The reduced boundary conditions are given by

$$
\begin{gathered}
f=0, f^{\prime}=1, \theta=1 \text { when } \eta=0 \\
f^{\prime} \rightarrow 0, \theta \rightarrow 0 \text { when } \eta \rightarrow \infty
\end{gathered}
$$

Where $F_{r}=\frac{C_{b}}{K^{\frac{1}{2}}}$ be an inertia coefficient, $\lambda=\frac{\nu}{\mathrm{aK}}$ be the porosity parameter, $R_{d}=\frac{16 \sigma T_{\infty}^{3}}{3 k k^{*}}$ be the radiation parameter and $\operatorname{Pr}=\frac{\mu c_{p}}{k}$ be the Prandtl number.

\section{Numerical Method}

In order to solve the boundary value problem (6)-(7) with boundary conditions (8), a shooting method consisted of Adams-Moulton methods is implemented. It is to be noted that some shooting methods are based on the use of Runge-Kutta methods which can give the accuracy of the order of four and five. Still, the explicit Runge-Kutta methods have the deficiency to meet stability condition. But the present class of constructed shooting methods is based on implicit methods which are called Adams-Moulton. But these implicit methods are coupled with Gauss-Seidel iterative method to solve the resulting difference equation, so the implementation is still considered as in an explicit manner. But this procedure of finding the solution using Adams-Moulton method(s) and Gauss-Seidel iterative method is required to find some initial conditions because Adams-Moulton method(s) are implemented on the system of first-order differential equations, so to solve the resulting difference equation, Gauss-Seidel iterative method is employed that requires the initial conditions for each of the equation in the system of differential equations. So to find the initial condition of each of the differential equation in the system, a shooting approach is considered that finds missing initial condition for each equation of the system. Also, it should be noted that some software packages may have the facility to find the solution using the shooting method, but this or these software packages use the RungeKutta methods to find the solution. Since all or most of the explicit methods are conditionally stable and may take more time to converge due to stability restriction but the present contribution is based on the use of implicit methods using Gauss-Seidel iterative method which are proved are unconditionally stable in next stability section for order two and three. Similarly, the stability requirement can be checked for higher-order Adams-Mouton methods coupled with the Gauss-Seidel iterative method.

Before applying Adams-Moulton methods, the considered boundary value problems are first reduced into the systems of first-order differential equations.

Using equations (6) and (7), the system of first-order differential equations can be expressed as:

Let $f^{\prime}=f_{1}(9)$

$f_{1}^{\prime}=f_{2}(10)$

$f_{2}^{\prime}=\lambda f_{1}+\left(1+F_{r}\right) f_{1}^{2}-f f_{2}(11)$

$\theta^{\prime}=\theta_{1}(12)$

$\theta_{1}^{\prime}=-\frac{P_{r}}{\beta} f \theta_{1}$

Where $\beta=1+\frac{4}{3} R_{d}(14)$

Since the present approach of applying numerical method is to solve discretized equations using Gauss-Seidel iterative method, the systems of equations (9)-(14) are linearized using Newton's linearization. So applying Newton's linearization on the nonlinear terms contained in equations (11) and (13)

$f_{1}^{2}=f_{1} f_{1}=f_{1} f_{1}^{k+1}+f_{1}^{k+1} f_{1}-f_{1} f_{1}=2 f_{1} f_{1}^{k+1}-f_{1}^{2}(15)$ 
$f f_{2}=f f_{2}^{k+1}+f^{k+1} f_{2}-f f_{2}(10)$

$f \theta_{1}=f \theta_{1}^{k+1}+f^{k+1} \theta_{1}-f \theta_{1}(17)$

Where the variables in bar notations in equations (15)-(17) are constants or previously computed iteration $k$. By using (15)-(17) into equations (11) and (14) and discretize the equations (9)-(14) using Adams-Moulton's second-order method the resulting equations are given by

$\frac{f_{i}-f_{i-1}}{h}=\frac{1}{2}\left(f_{1, i}+f_{1, i-1}\right)(18)$

$\frac{f_{1, i}-f_{1, i-1}}{h}=\frac{1}{2}\left(f_{2, i}+f_{2, i-1}\right)(19)$

$\frac{f_{2, i}-f_{2, i-1}}{h}=\frac{1}{2}\left(\lambda f_{1, i}+\left(1+F_{r}\right)\left(2 f_{1, i} f_{1, i}-f_{1, i}^{2}\right)-f_{i} f_{2, i}-f_{i} f_{2, i}+f_{i} f_{2, i}+\lambda f_{1, i-1}+\left(1+F_{r}\right)\left(2 f_{1} f_{1, i-1}-f_{1, i-1}^{2}\right)-\right.$

$\left.f_{i-1} f_{2, i-1}-f_{i-1} f_{2, i-1}+f_{i-1} f_{2, i-1}\right)(20)$

$\frac{\theta_{i-} \theta_{i-1}}{h}=\frac{1}{2}\left(\theta_{1, i}+\theta_{1, i-1}\right)(21)$

$\frac{\theta_{1, i-} \theta_{1, i-1}}{h}=\frac{1}{2}\left[-\frac{P_{r}}{\beta}\left(f_{i} \theta_{1, i}+f_{i} \theta_{1, i}-f_{i} \theta_{1, i}+f_{i-1} \theta_{1, i-1}+f_{i-1} \theta_{1, i-1}-f_{i-1} \theta_{1, i-1}\right)\right](22)$

By applying the Gauss-Seidel iterative method on equations (18)-(22) the resulting equations are expressed by

$\frac{f_{i}^{k+1}-f_{i-1}^{k+1}}{h}=\frac{1}{2}\left(f_{1, i}^{k}+f_{1, i-1}^{k+1}\right)(23)$

$\frac{f_{1, i}^{k+1}-f_{1, i-1}^{k+1}}{h}=\frac{1}{2}\left(f_{2, i}^{k}+f_{2, i-1}^{k+1}\right)(24)$

$\frac{f_{2, i}^{k+1}-f_{2, i-1}^{k+1}}{h}=\frac{1}{2}\left(\lambda f_{1, i}^{k+1}+\left(1+F_{r}\right)\left(2 f_{1, i}^{k} f_{1, i}^{k+1}-\left(f_{1, i}^{k}\right)^{2}-f_{i}^{k} f_{2, i}^{k}-f_{i}^{k+1} f_{2, i}^{k}+f_{i}^{k} f_{2, i}^{k}+\left(1+F_{r}\right)\left(2 f_{1, i-1}^{k} f_{1, i-1}^{k+1}-\left(f_{1, i-1}^{k}\right)^{2}\right)-\right.\right.$

$\left.f_{i-1}^{k} f_{2, i-1}^{k+1}-f_{i}^{k+1} f_{2, i-1}^{k}+f_{i-1}^{k} f_{2, i-1}^{k}\right)(25)$

$\frac{\theta_{i}^{k+1}-\theta_{i-1}^{k+1}}{h}=\frac{1}{2}\left(\theta_{1, i}^{k}+\theta_{1, i-1}^{k+1}\right)(26)$

$\frac{\theta_{1, i}^{k+1}-\theta_{1, i-1}^{k+1}}{h}=\frac{P_{r}}{2 \beta}\left(f_{i}^{k} \theta_{1, i}^{k}+f_{i}^{k+1} \theta_{1, i}^{k}-f_{i}^{k} \theta_{1, i}^{k}+f_{i-1}^{k} \theta_{1, i-1}^{k+1}+f_{i-1}^{k+1} \theta_{1, i-1}^{k}-f_{i-1}^{k} \theta_{1, i-1}^{k}\right)(27)$

Where $h$ be a fixed step size of the considered finite difference domain.

\section{Stability Analysis}

In order to check the stability criteria of resulting equations (23)-(27), reduce the system into the following vector-matrix form

$X^{\prime}=A X(28)$

Where $X=$

$\left[\begin{array}{c}f \\ f_{1} \\ f_{2} \\ \theta\end{array}\right]$ and $A=$

$\left[\begin{array}{ccccc}0 & a m p ; 1 & a m p ; 0 & a m p ; 0 & a m p ; 0 \\ 0 & a m p ; 0 & a m p ; 1 & a m p ; 0 & a m p ; 0 \\ -f_{2} & a m p ; \lambda+\left(1+F_{r} 2 f_{1}\right) & a m p ;-f & a m p ; 0 & a m p ; 0 \\ 0 & a m p ; 0 & a m p ; 0 & a m p ; 0 & a m p ; 1 \\ -\frac{P_{r}}{\beta} \theta_{1} & a m p ; 0 & a m p ; 0 & a m p ; 0 & a m p ;-\frac{P_{r}}{\beta} f\end{array}\right]$

By applying the Adams-Moulton's second-order method on equation (28) yields 
$\frac{X_{i}-X_{i-1}}{h}=\frac{A}{2}\left(X_{i}+X_{i-1}\right)$

By applying Gauss-Seidel iterative method on equation (29) gives the following equation

$\frac{X_{i}^{k+1}-X_{i-1}^{k+1}}{h}=\frac{A}{2}\left(X_{i}^{k}+X_{i-1}^{k+1}\right)(30)$

In the present contribution, the Von Neuman stability criteria are employed. The criteria are usually applied to partial differential equations. Still, in this contribution, it is applied on ordinary differential equation with discretisation is given using Adams- Moulton second-order method and iterative procedure of Gauss-Seidel iterative method.

Substitution of $X_{i}^{k+1}=\phi^{k+1} e^{\iota \mathrm{I}}, X_{i}^{k}=\phi^{k} e^{\iota \mathrm{I}}, X_{i-1}^{k+1}=\phi^{k+1} e^{(i-1) I \phi}$ into equation (30), the resulting equation is given by

$$
\underline{\phi^{k+1} e^{\mathrm{II}}-\phi^{k+1} e^{(i-1) I \phi}}
$$

englishh $=\mathrm{A} \frac{}{2\left(\phi^{k} e^{\mathrm{II}}+\phi^{k+1} e^{(i-1) I \phi}\right)}$

Dividing the whole equation by $e^{\mathrm{\iota I}}$

$$
\begin{gathered}
\frac{\phi^{k+1}-\phi^{k+1} e^{-I \phi}}{h}=\frac{A}{2}\left(\phi^{k}+\phi^{k+1} e^{-I \phi}\right) \\
\left(1 . D-e^{-I \phi} 1 . D-\frac{h}{2} A\right) \phi^{k+1}=\frac{h}{2} A \phi^{k}
\end{gathered}
$$

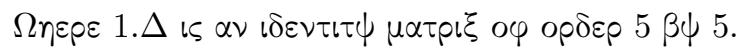

$G=\frac{\phi^{k+1}}{\phi^{k}}=\left(1 . D-e^{-I \phi} 1 . D-\frac{h}{2} A\right)^{-1}\left(\frac{h}{2} A\right)$

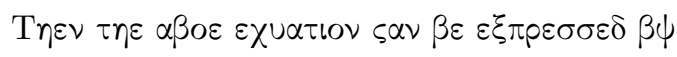

$G=\left(1-e^{-I \phi}-\frac{h}{2} \lambda_{\max }\right)^{-1}\left(\frac{h}{2} \lambda_{\max }\right)$

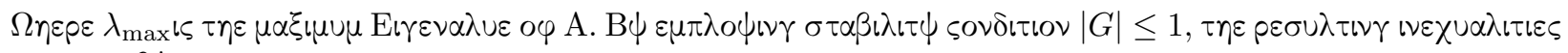

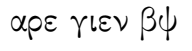

$\left|\frac{h}{2}\left(1-e^{-I \phi}-\frac{h}{2} \lambda_{\max }\right)^{-1} \lambda_{\max }\right| \leq 1$

$\frac{h}{2}\left|\lambda_{\max }\right| \leq\left|1-e^{-I \phi}-\frac{h}{2} \lambda_{\max }\right| \leq 1+1+\frac{h}{2}\left|\lambda_{\max }\right|$

$\frac{h}{2}\left|\lambda_{\max }\right| \leq 2+\frac{h}{2}\left|\lambda_{\max }\right|$

$0 \leq 2$

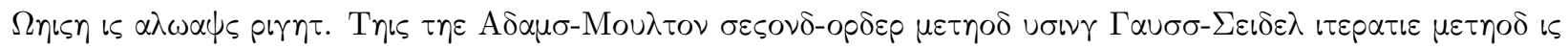

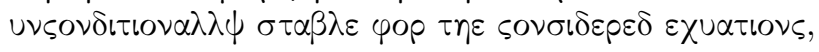

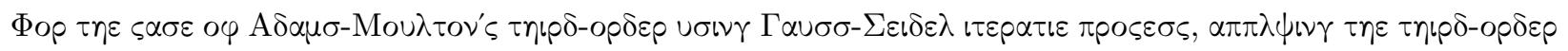

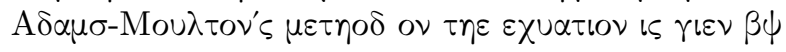

$\frac{X_{i}-X_{i-1}}{h}=\frac{A}{12}\left(5 X_{i}+8 X_{i-1}-X_{i-2}\right)(31)$

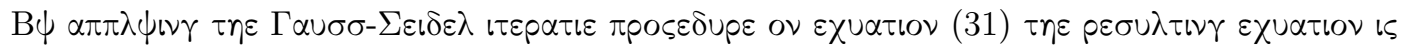

$\frac{X_{i}^{k+1}-X_{i-1}^{k+1}}{h}=\frac{A}{12}\left(5 X_{i}^{k}+8 X_{i-1}^{k+1}-X_{i-2}^{k+1}\right)(32)$ 


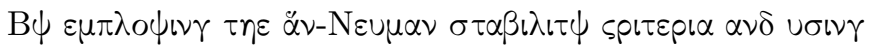

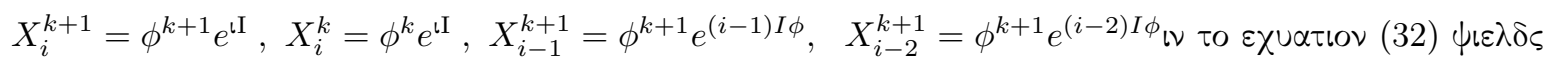

$\underline{\phi^{k+1} e^{i I}-\phi^{k+1} e^{(i-1) I \phi}}$

$\varepsilon \vee \gamma \lambda เ \sigma \eta \eta=\mathrm{A} \frac{}{12\left(5 \phi^{k} e^{l I}+8 \phi^{k+1} e^{(i-1) I}-\phi^{k+1} e^{(i-2) I \phi)}\right.}(33)$

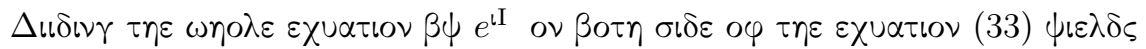

$$
\begin{gathered}
\frac{\phi^{k+1}-\phi^{k+1} e^{-I \phi}}{h}=\frac{A}{12}\left(5 \phi^{k}+8 \phi^{k+1} e^{-I \phi}-\phi^{k+1} e^{-2 I \phi}\right) \\
\left(1 . D-e^{-I \phi} 1 . D-\frac{2 h}{3} A e^{-I \phi}+\frac{h}{12} A e^{-2 I \phi}\right) \phi^{k+1}=\frac{5 h}{12} A \phi^{k}
\end{gathered}
$$

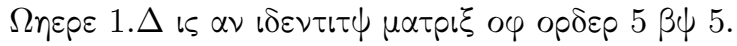

$G=\frac{\phi^{k+1}}{\phi^{k}}=\left(1 . D-e^{-I \phi} 1 . D-\frac{2 h}{3} e^{-I \phi} A+\frac{h}{12} e^{-2 I \phi} A\right)^{-1}\left(\frac{5 h}{12} A\right)$

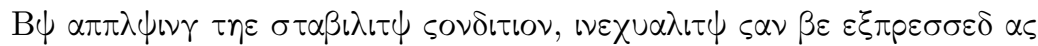

$$
\left|\left(1-e^{-I \phi}-\frac{2}{3} h e^{-I \phi} \lambda_{\max }+\frac{h}{12} e^{-2 I \phi} \lambda_{\max }\right)^{-1} \frac{5 h}{12} \lambda_{\max }\right| \leq 1
$$

$\frac{5 h}{2}\left|\lambda_{\max }\right| \leq\left|1-e^{-I \phi}-\frac{2}{3} h e^{-I \phi} \lambda_{\max } \frac{h}{12} e^{-2 I \phi} \lambda_{\max +}\right| \leq 1+1+\frac{2}{3} h\left|\lambda_{\max }\right|+\frac{h}{12}\left|\lambda_{\max }\right|$

$\frac{h}{12}(5-8-1)\left|\lambda_{\max }\right| \leq 2$

$\left|\lambda_{\max }\right| \geq-\frac{6}{h}$

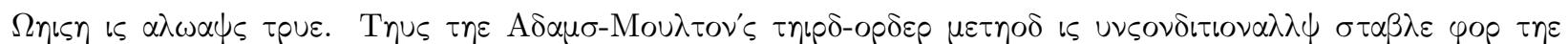

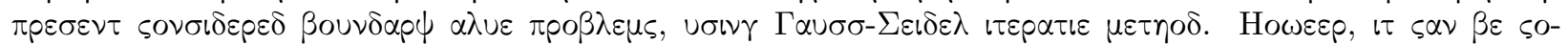

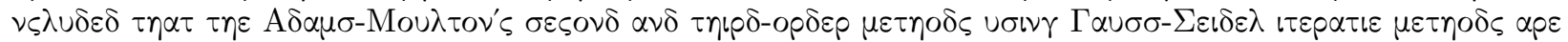

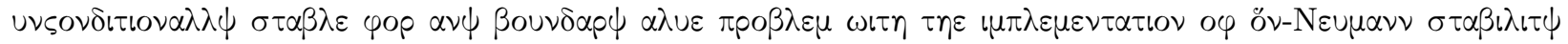

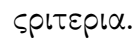

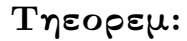

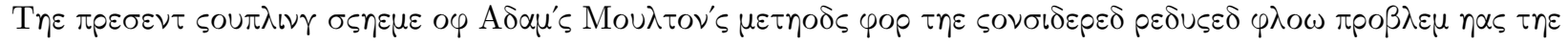

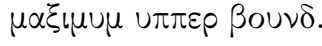

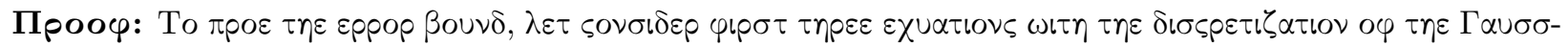
$\Sigma \varepsilon เ \delta \varepsilon \lambda \mu \varepsilon \tau \eta\rceil \delta$

$$
\begin{aligned}
& \frac{f_{i}^{k+1}-f_{i-1}^{k+1}}{h}=\frac{1}{2}\left(f_{1, i}^{k}+f_{1, i-1}^{k+1}\right)(34) \\
& \frac{f_{1, i}^{k+1}-f_{1, i-1}^{k+1}}{h}=\frac{1}{2}\left(f_{2, i}^{k}+f_{2, i-1}^{k+1}\right)(35) \\
& \frac{f_{2, i}^{k+1}-f_{2, i-1}^{k+1}}{h}=\frac{1}{2}\left(\lambda\left(f_{1, i}^{k+1}+f_{1, i-1}^{k+1}\right)+\left(1+F_{r}\right)\left(2 f_{1} f_{1, i}^{k+1}+2 f_{1} f_{1, i-1}^{k+1}-f_{1}^{2}\right)-f\left(f_{2, i}^{k}+f_{2, i-1}^{k+1}\right)-f_{2}\left(f_{i}^{k+1}+f_{i-1}^{k+1}\right)+\right.
\end{aligned}
$$

$\left.f f_{2}\right)(36)$

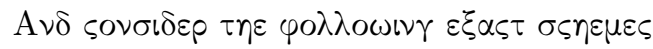

$$
\frac{f_{i}^{E}-f_{i-1}^{E}}{h}=\frac{1}{2}\left(f_{1, i}^{E}+f_{1, i-1}^{E}\right)(37)
$$


$\frac{f_{1, i}^{E}-f_{1, i-1}^{E}}{h}=\frac{1}{2}\left(f_{2, i}^{E}+f_{2, i-1}^{E}\right)(38)$

$\frac{f_{2, i}^{E}-f_{2, i-1}^{E}}{h}=\frac{1}{2}\left(\lambda\left(f_{1, i}^{E}+f_{1, i-1}^{E}\right)+\left(1+F_{r}\right)\left(2 f_{1} f_{1, i}^{E}+2 f_{1} f_{1, i-1}^{E}-f_{1}^{2}\right)-f\left(f_{2, i}^{E}+f_{2, i-1}^{E}\right)-f_{1}\left(f_{i}^{E}+f_{i-1}^{E}\right)+\right.$ $\left.f f_{2}\right)(39)$

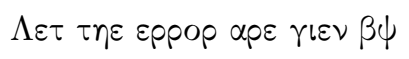

$$
e_{1, i}^{k}=f_{i}^{k}-f_{i}^{E}, e_{2, i}^{k}=f_{1, i}^{k}-f_{1, i}^{E}, e_{3, i}^{k}=f_{2, i}^{k}-f_{2, i}^{E}
$$

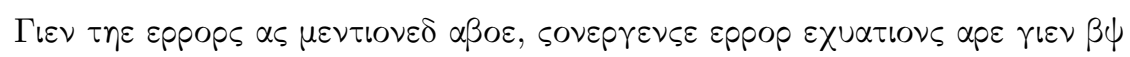

$\frac{e_{1, i}^{k+1}-e_{1, i-1}^{k+1}}{h}=\frac{1}{2}\left(e_{2, i}^{k}+e_{2, i-1}^{k+1}\right)(40)$

$\frac{e_{2, i}^{k+1}-e_{2, i-1}^{k+1}}{h}=\frac{1}{2}\left(e_{3, i}^{k}+e_{3, i-1}^{k+1}\right)(41)$

$\frac{e_{3, i}^{k+1}-e_{3, i-1}^{k+1}}{h}=\frac{1}{2}\left(\lambda\left(e_{2, i}^{k+1}+e_{2, i-1}^{k+1}\right)+\left(1+F_{r}\right)\left(2 f_{1} e_{2, i}^{k+1}+2 f_{1} e_{2, i-1}^{k+1}\right)-f\left(e_{3, i}^{k}+e_{3, i-1}^{k+1}\right)-f_{2}\left(e_{1, i}^{k+1}+e_{1, i-1}^{k+1}\right)\right)($

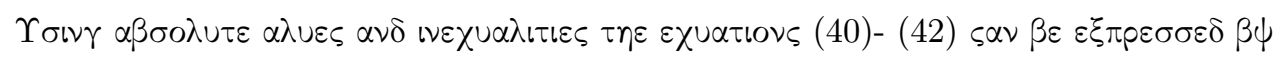

$\left|e_{2, i-1}^{k+1}\right| \leq\left|e_{2,1}^{k}\right|+\frac{2}{h}\left(\left|e_{1, i-1}^{k+1}\right|\right)+\left|e_{1, i}^{k+1}\right|+M_{1} \theta\left(h^{2}\right)(43)$

$\left|e_{3, i-1}^{k+1}\right| \leq\left|e_{3,1}^{k}\right|+\frac{2}{h}\left(\left|e_{2, i-1}^{k+1}\right|\right)+\left|e_{2, i}^{k+1}\right|+M_{2} \theta\left(h^{2}\right)(44)$

$\left|e_{3, i}^{k+1}\right|+\left|e_{3, i-1}^{k+1}\right| \geq \frac{h}{2}\left[-|\lambda|\left(\left|e_{2, i}^{k+1}\right|+\left|e_{2, i-1}^{k+1}\right|\right)-\left(1+F_{r}\right) 2 f_{1}\left(\left|e_{2, i}^{k+1}\right|+\left|e_{2, i-1}^{k+1}\right|\right)-|f|\left(\left|e_{3, i}^{k}\right|+\left|e_{3, i-1}^{k+1}\right|\right)+\left|f_{2}\right|\left(\left|e_{1, i}^{k+1}\right|+\left|e_{1, i-1}^{k+1}\right|\right)\right]+$ $M_{3} \theta\left(h^{2}\right)(45)$

$\Lambda \varepsilon \tau e_{1}^{k}=\max _{1 \leq i \leq N}\left|e_{1, i}^{k}\right|, e_{2}^{k}=\max _{1 \leq i \leq N}\left|e_{2, i}^{k}\right|, e_{3}^{k}=\max _{1 \leq i \leq N}\left|e_{3, i}^{k}\right|$

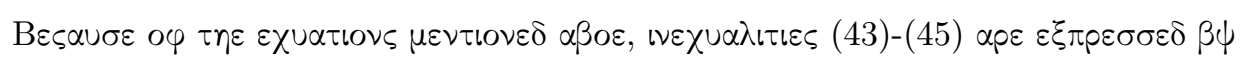

$e_{2}^{k+1} \leq e_{2}^{k}+\frac{2}{h}\left(2 e_{1}^{k+1}\right)+M_{1} \theta\left(h^{2}\right)(46)$

$e_{3}^{k+1} \leq e_{3}^{k}+\frac{2}{h}\left(2 e_{2}^{k+1}\right)+M_{2} \theta\left(h^{2}\right)(47)$

$\frac{h}{2}\left|f_{2}\right| 2 e_{1}^{k+1} \leq \frac{2}{h}\left(2 e_{3}^{k+1}+|\lambda| 2 e_{2}^{k+1}+\left|1+F_{r}\right| 2 f_{1} 2 e_{2}^{k+1}+|f|\left(e_{3}^{k}+e_{3}^{k+1}\right)\right)$

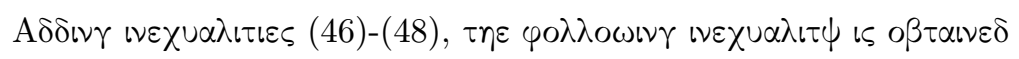

$\frac{h}{2}\left|f_{2}\right| 2 e_{1}^{k+1}+e_{2}^{k+1}+e_{3}^{k+1} \leq e_{2}^{k}+\frac{4}{h} e_{1}^{k+1}+e_{3}^{k}+\frac{4}{h} e_{2}^{k+1}+\frac{4}{h} e_{3}^{k+1}+\frac{h}{2}\left[|\lambda| 2 e_{2}^{k+1}+\left|1+F_{r}\right| 4 f_{1} e_{2}^{k+1}+|f|\left(e_{3}^{k}+\right.\right.$ $\left.\left.e_{3}^{k+1}\right)\right]+M \theta\left(h^{2}\right)(49)$

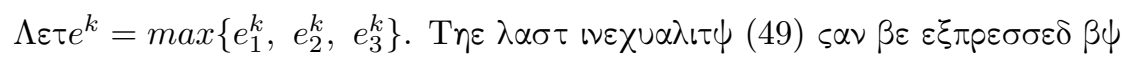

$\frac{h}{2}\left|f_{2}\right| 2 e^{k+1}+e^{k+1}+e^{k+1} \leq e^{k}+\frac{4}{h} e^{k+1}+e^{k}+\frac{4}{h} e^{k+1}+\frac{4}{h} e^{k+1}+\frac{h}{2}\left[|\lambda| 2 e^{k+1}+\left|1+F_{r}\right| 4\left|f_{1}\right| e^{k+1}+|f|\left(e^{k}+\right.\right.$ $\left.\left.e^{k+1}\right)\right]+M \theta\left(h^{2}\right)$

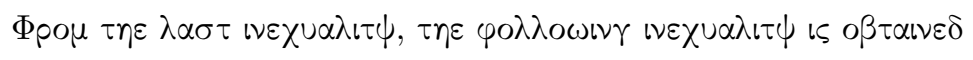

$h\left|f_{2}\right|+2-\frac{4}{h}-\frac{4}{h}-\frac{4}{h}-h|\lambda|-h\left|1+F_{r}\right| 2\left(\left|f_{1}\right|-\frac{h}{2}|f|\right) e^{k+1} \leq 2 e^{k}+\frac{h}{2}|f| e^{k}+M \theta\left(h^{2}\right)(50)$

$\Lambda \varepsilon \tau \beta=h\left|f_{2}\right|+2-\frac{12}{h}-h|\lambda|-h\left|1+F_{r}\right| 2\left|f_{1}\right|-\frac{h}{2}|f|$

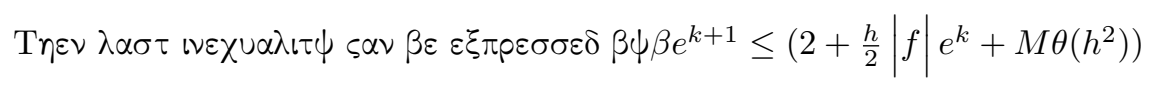


$\Lambda \varepsilon \tau \alpha=2+\frac{h}{2}|f|$

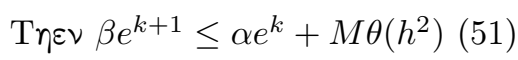

$\Lambda \varepsilon \tau k=0$

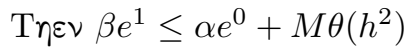

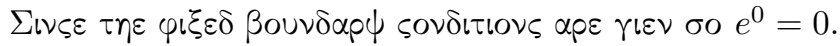

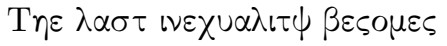

$\beta e^{1} \leq+M \theta\left(h^{2}\right)$

$\Lambda \varepsilon \tau k=1 \iota(51)$

$$
\begin{aligned}
\beta e^{2} & \leq \alpha e^{1}+M \theta\left(h^{2}\right) \\
e^{2} & \leq \frac{\alpha}{\beta} e^{1}+\frac{M}{\beta} \theta\left(h^{2}\right)
\end{aligned}
$$

$\Lambda \varepsilon \tau \frac{M}{\beta}=M_{\beta}, \alpha \nu \delta \frac{\alpha}{\beta}=\gamma$

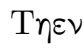

$e^{2} \leq \gamma e^{1}+M_{\beta} \theta\left(h^{2}\right) \leq \gamma\left(M_{\beta} \theta\left(h^{2}\right)\right)+M_{\beta} \theta\left(h^{2}\right)=(\gamma+1) M_{\beta} \theta\left(h^{2}\right)$

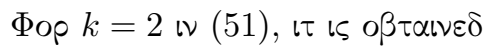

$e^{3} \leq \gamma e^{2}+M_{\beta} \theta\left(h^{2}\right) \leq \gamma(\gamma+1)\left(M_{\beta} \theta\left(h^{2}\right)\right)+M_{\beta} \theta\left(h^{2}\right)=\left(\gamma^{2}+\gamma+1\right) M_{\beta} \theta\left(h^{2}\right)$

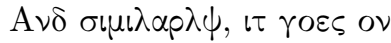

$e^{k} \leq\left(1+r+r^{2}+\ldots+r^{k-1}\right) M_{\beta} \theta\left(h^{2}\right)=\frac{1-r^{k}}{1-r} M_{\beta} \theta\left(h^{2}\right)$

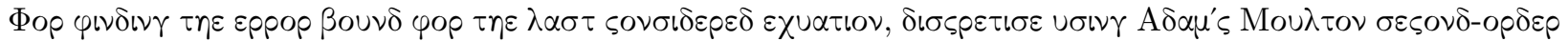

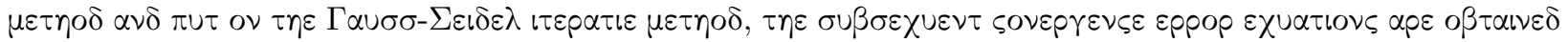

$\frac{e_{4, i}^{k+1}-e_{4, i-1}^{k+1}}{h}=\frac{1}{2}\left(e_{5, i}^{k+1}+e_{4, i-1}^{k+1}\right)(52)$

$\frac{e_{5, i}^{k+1}-e_{5, i-1}^{k+1}}{h}=-\frac{P_{r}}{2\left(1+\frac{4}{3} R_{d}\right)}\left[f\left(e_{5, i}^{k+1}-e_{5, i-1}^{k+1}\right)+\theta_{1}\left(e_{1, i}^{k+1}-e_{1, i-1}^{k+1}\right)\right]$

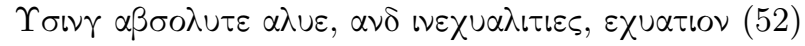

$\left|e_{5, i-1}^{k+1}\right| \leq\left|e_{5, i}^{k+1}\right|+\frac{2}{h}\left(\left|e_{4, i}^{k+1}\right|-\left|e_{4, i-1}^{k+1}\right|\right)+M_{4} \theta\left(h^{2}\right)(54)$

$\frac{2}{h} e_{5}^{k+1} \geq \frac{\delta}{2}\left[|f| e_{5}^{k+1}-|f| e_{5}^{k}-\left|\theta_{1}\right| 2 e_{1}^{k+1}\right]$

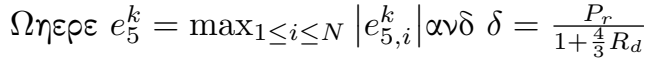

$|f| e_{5}^{k+1} \leq \frac{4}{\eta \tilde{\delta}} e_{5}^{k+1}+|f| e_{5}^{k}+2\left|\theta_{1}\right| e_{1}^{k+1}+M_{5} \theta\left(h^{2}\right)$

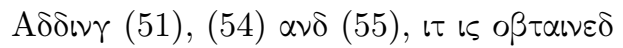

$|f| e_{5}^{k+1}+e^{k+1}+e_{5}^{k+1} \leq \frac{4}{\eta \tilde{\delta}} e_{5}^{k+1}+|f| e_{5}^{k}+2\left|\theta_{1}\right| e_{1}^{k+1}+\gamma e^{k}+M \theta\left(h^{2}\right)+e_{5}^{k}+\frac{4}{h} e_{4}^{k+1} M_{5} \theta\left(h^{2}\right)$

$\Lambda \varepsilon \tau e^{-k}=\max \left\{e_{5}^{k}, e^{k}, e_{4}^{k}\right\}$ 


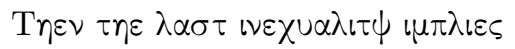

$$
\left(|f|+2-\frac{4}{\eta \delta}-2\left|\theta_{1}\right|-\frac{4}{h}\right) e^{-k+1} \leq|f| e^{-k}+\gamma e^{-k}+e^{-k}+M_{5} \theta\left(h^{2}\right)
$$

$\Lambda \varepsilon \tau \delta_{1}=|f|+2-\frac{4}{\eta \tilde{\delta}}-2\left|\theta_{1}\right|-\frac{4}{h} \alpha \nu \delta \delta_{2}=|f|+\gamma+1$

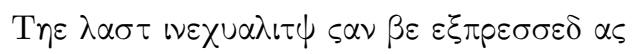

$\delta_{1} e^{-k+1} \leq \delta_{2} e^{-k}+M_{5} \theta\left(h^{2}\right)$

$\Lambda \varepsilon \tau \frac{\delta_{2}}{\delta_{1}}=\delta_{3} \alpha \nu \delta \frac{M_{5}}{\delta_{1}}=M_{\delta}$

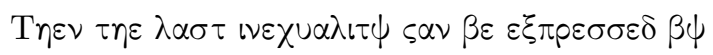

$e^{-k+1} \leq \delta_{3} e^{-k}+M_{\delta} \theta\left(h^{2}\right)(56)$

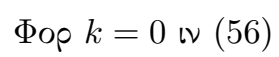

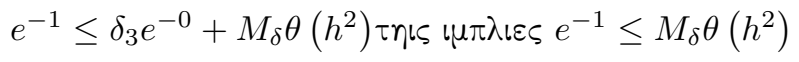

Фор $k=1$ เ (56)

$e^{-2} \leq \delta_{3} e^{-1}+M_{\delta} \theta\left(h^{2}\right) \leq \delta_{3} M_{\delta} \theta\left(h^{2}\right)+M_{\delta} \theta\left(h^{2}\right)=\left(\delta_{3}+1\right) M_{\delta} \theta\left(h^{2}\right)$

Фор $k=2$ เ (56)

$e^{-3} \leq \delta_{3} e^{-2}+M_{\delta} \theta\left(h^{2}\right) \leq \delta_{3}\left(\delta_{3}+1\right) M_{\delta} \theta\left(h^{2}\right)+M_{\delta} \theta\left(h^{2}\right)=\left(\delta_{3}{ }^{2}+\delta_{3}+1\right) M_{\delta} \theta\left(h^{2}\right)$

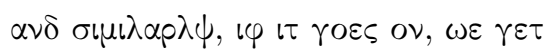

$$
e^{-k} \leq\left(1+\delta_{3}+\delta_{3}{ }^{2}+\ldots+\delta_{3}{ }^{k-1}\right) M_{\delta} \theta\left(h^{2}\right)=\frac{1-\delta_{3}{ }^{k}}{1-\delta_{3}} M_{\delta} \theta\left(h^{2}\right)
$$

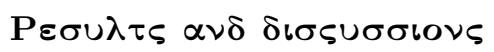

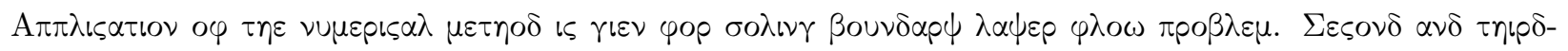

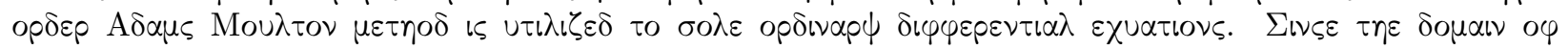

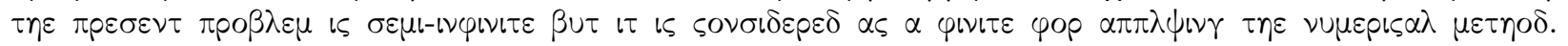

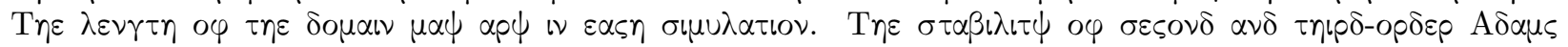

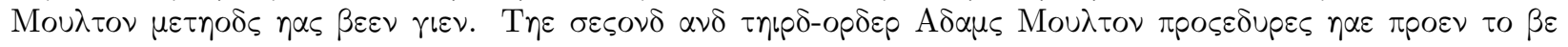

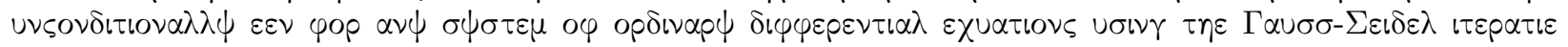

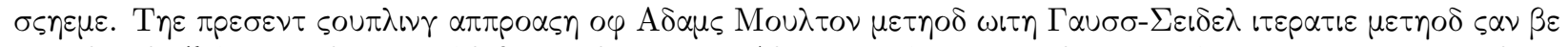

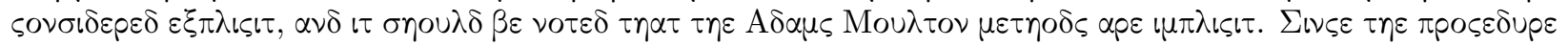

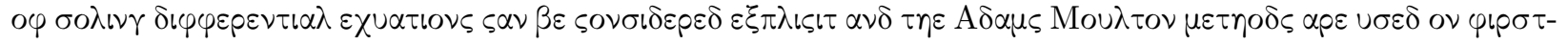

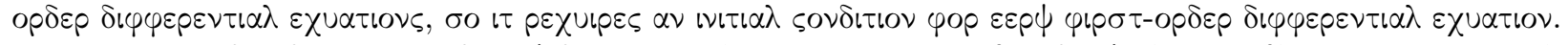

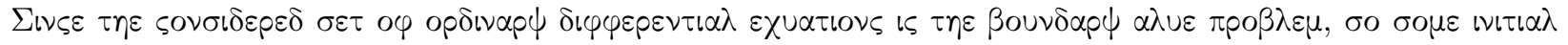

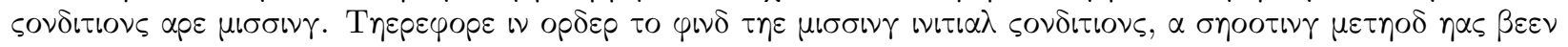

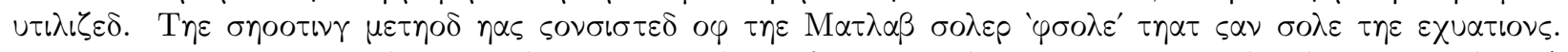

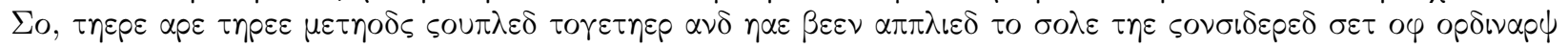

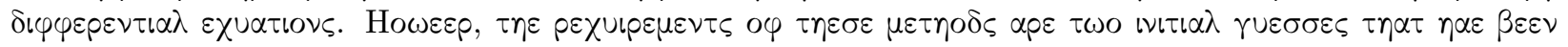

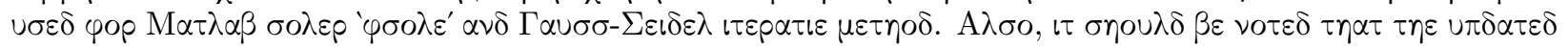

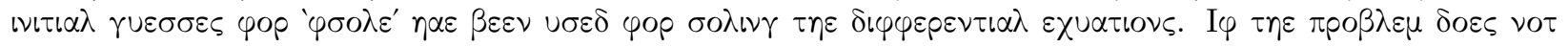

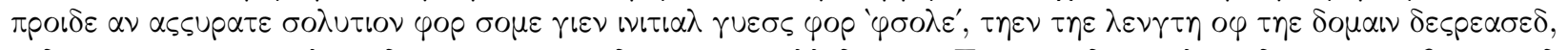

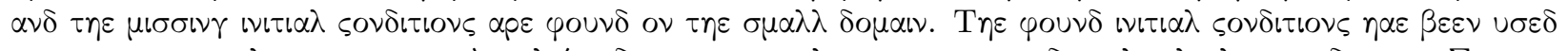

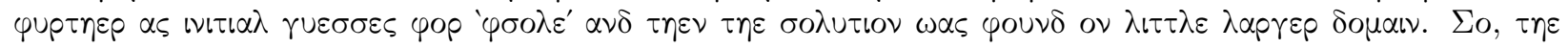




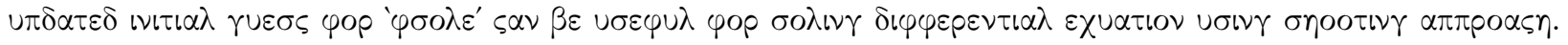

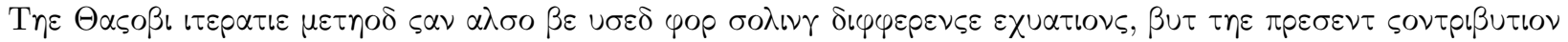
is $\delta \varepsilon \alpha \lambda \tau \omega เ \tau \eta \eta \eta \varepsilon \Gamma \alpha u \sigma \sigma-\Sigma \varepsilon\llcorner\delta \varepsilon \lambda \mu \varepsilon \tau \eta о \delta$.

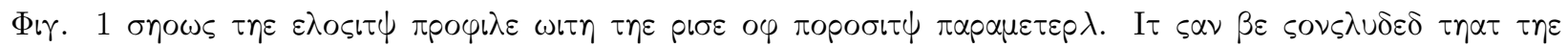

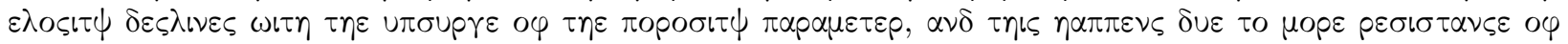

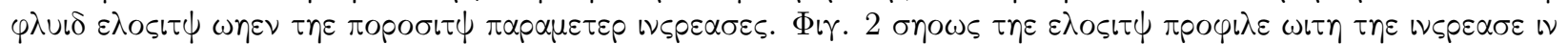

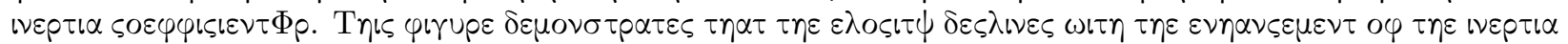

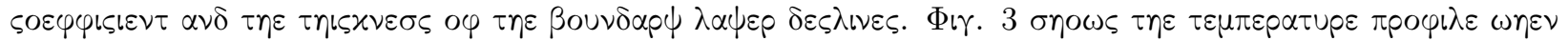

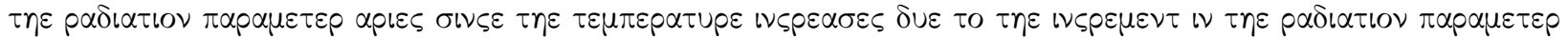

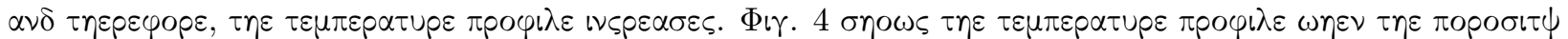

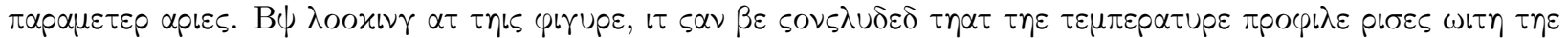

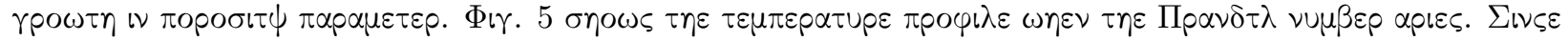

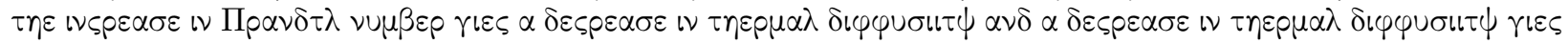

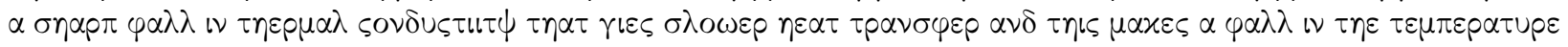
$\pi \rho \circ \iota \iota \lambda \varepsilon$.

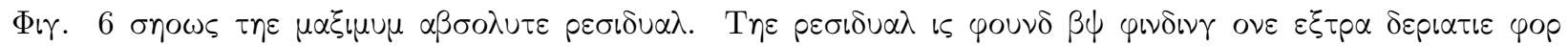

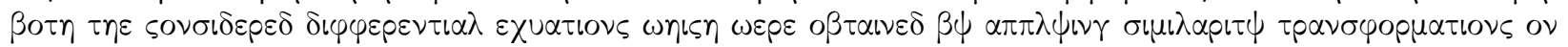

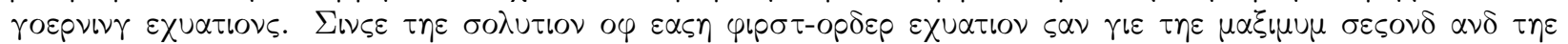

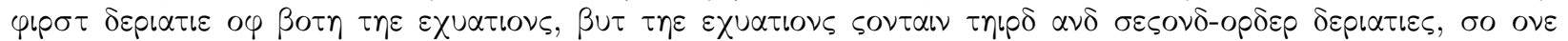

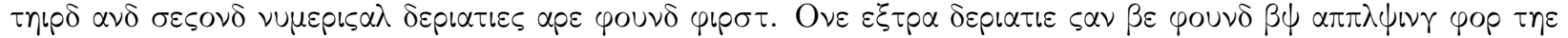

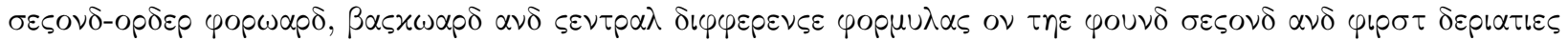

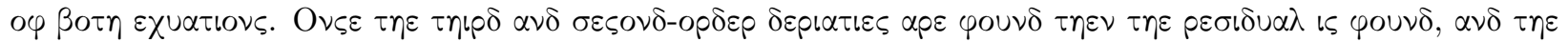

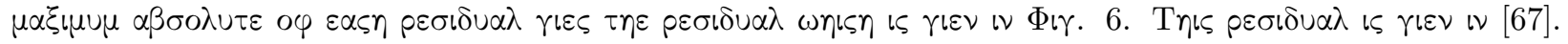

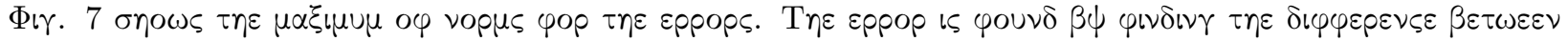

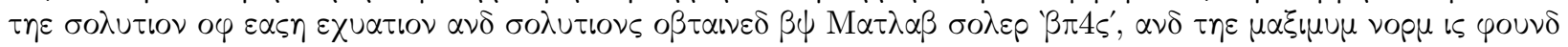

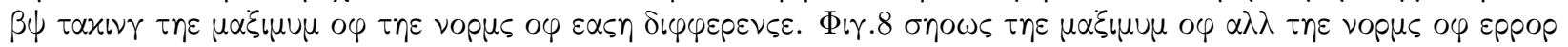

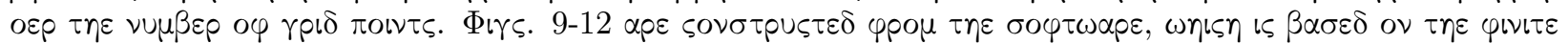

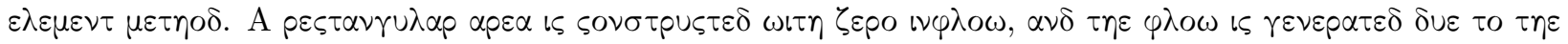

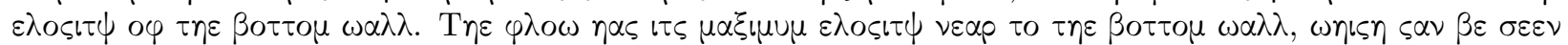

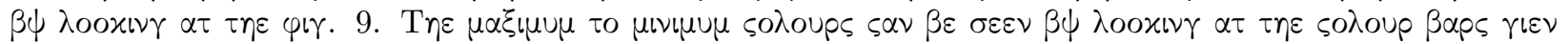

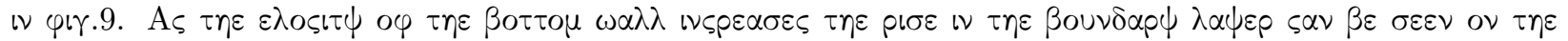

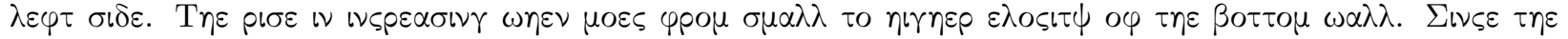

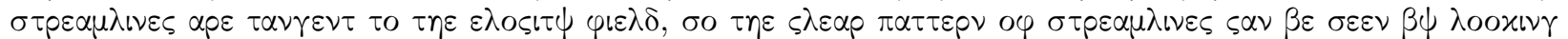

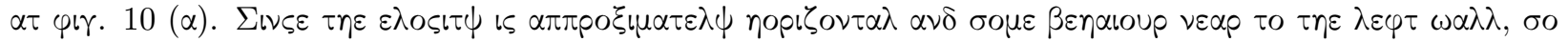

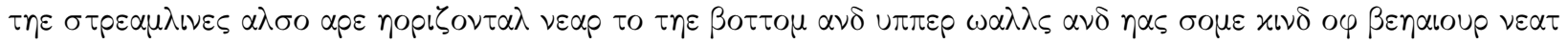

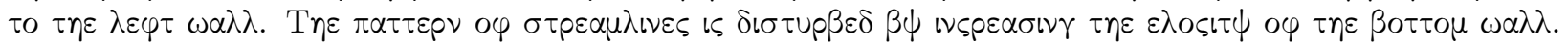

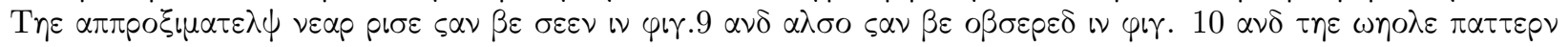

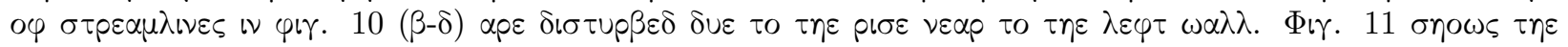

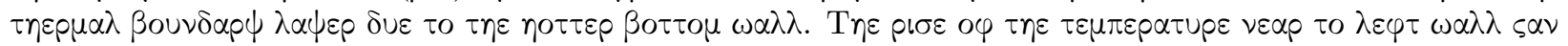

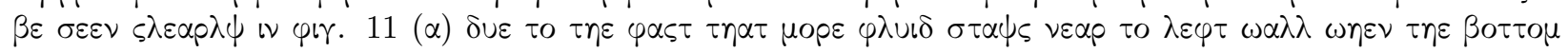

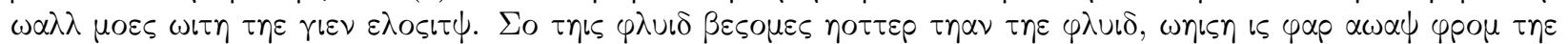

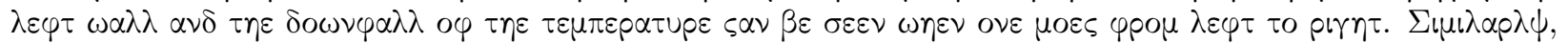
$\tau \eta \varepsilon \tau \varepsilon \mu \pi \varepsilon \rho \alpha \tau$

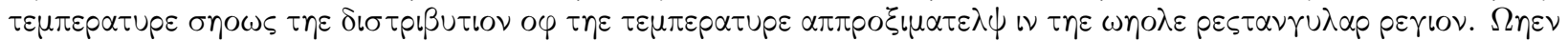

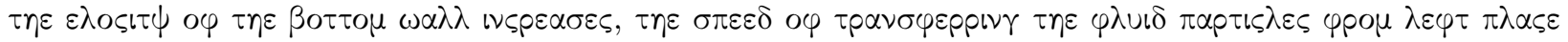

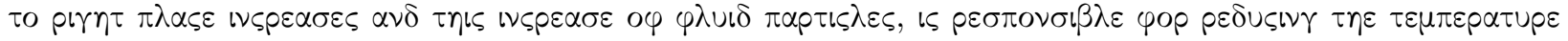

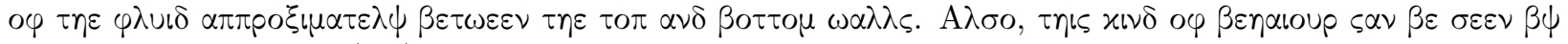

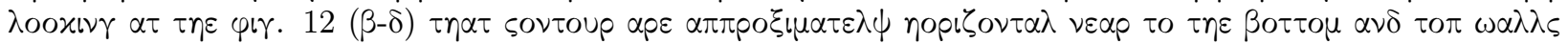

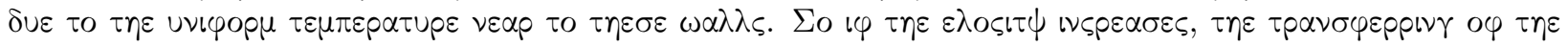

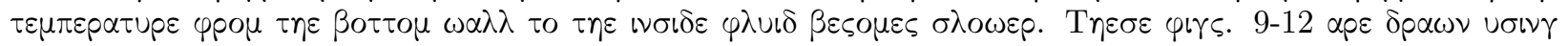




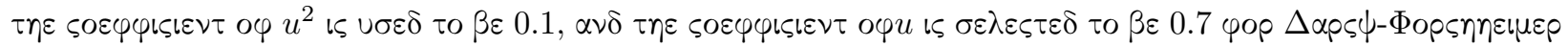


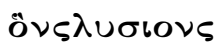

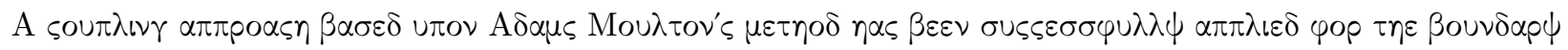

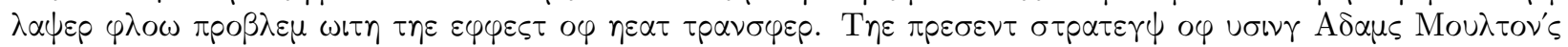

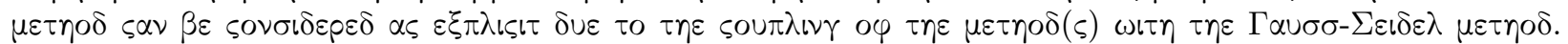

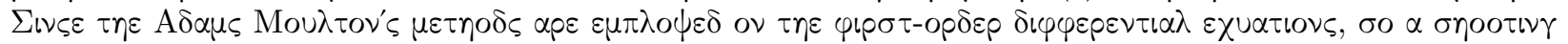

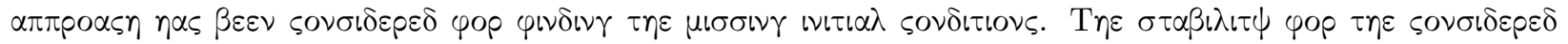

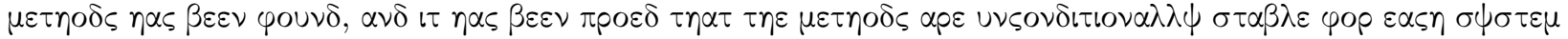

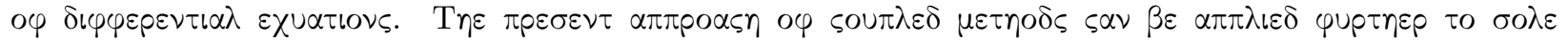
$\delta\llcorner\varphi \varphi \varepsilon p \varepsilon \nu \tau \iota \alpha \lambda \varepsilon \chi \cup \alpha \tau \iota$

\section{A $\varsigma \nu \nu \omega \lambda \varepsilon \delta \gamma \varepsilon \mu \varepsilon \nu \tau \varsigma$}

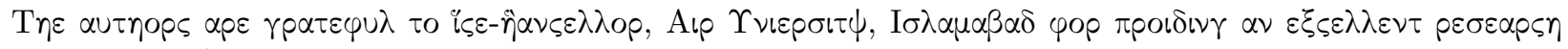

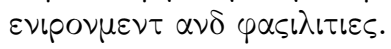

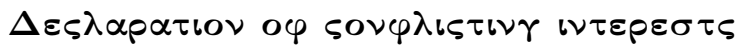

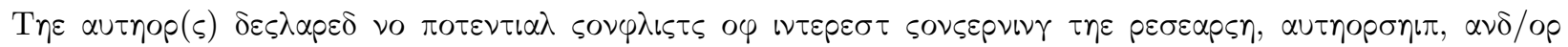

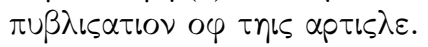

\section{$\Phi \cup \nu \delta\llcorner\gamma$}

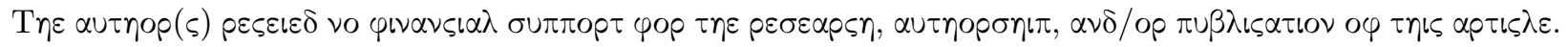

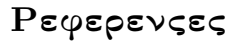

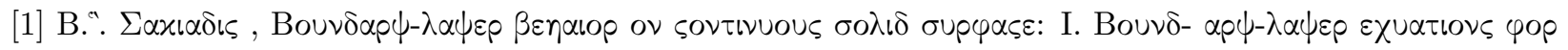

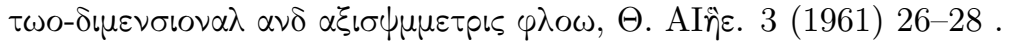

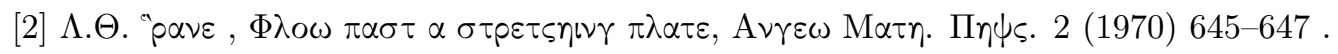

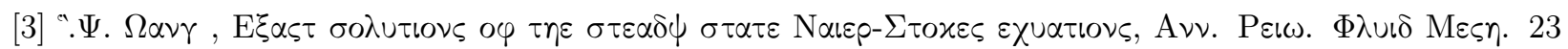
(1991) 159-177 .

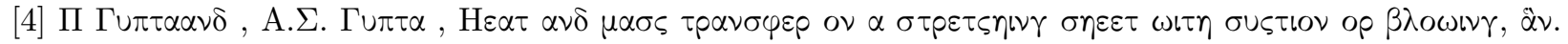

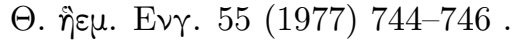

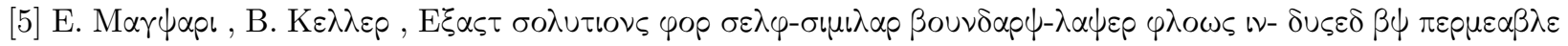

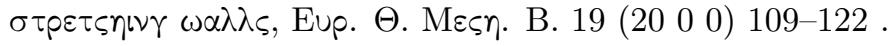

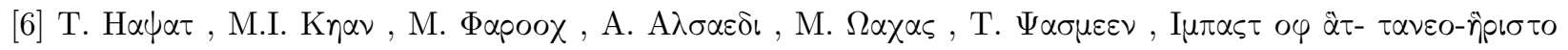

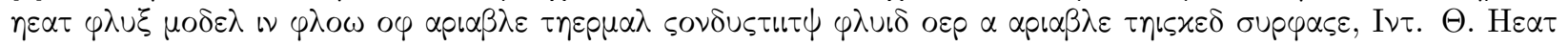

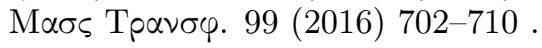

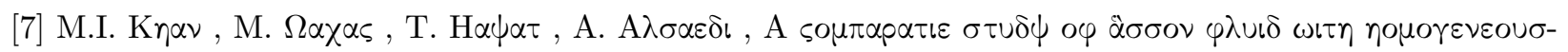

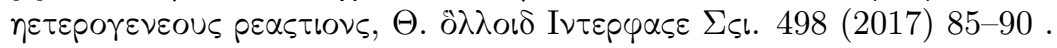

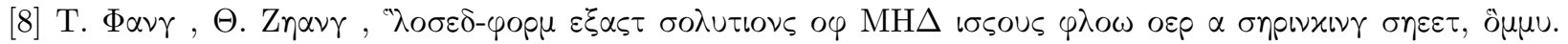

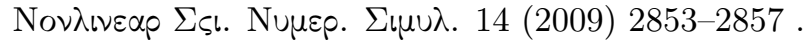

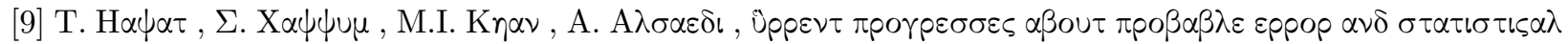

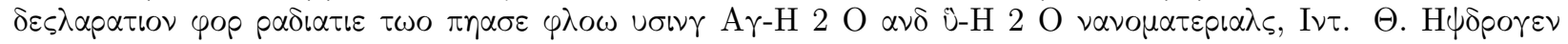
Eveprభ 42 (2017) 29107-29120 .

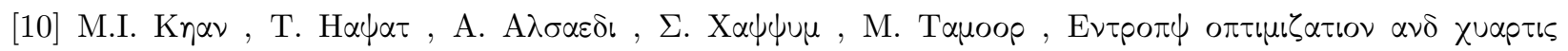

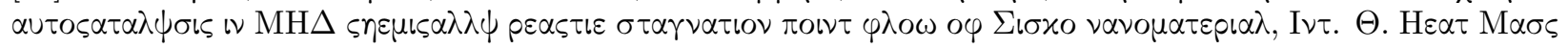

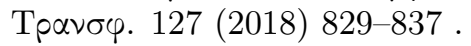




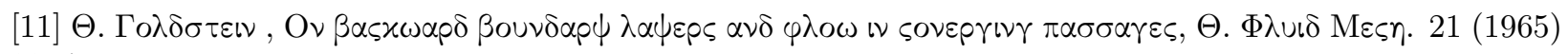
$33-45$.

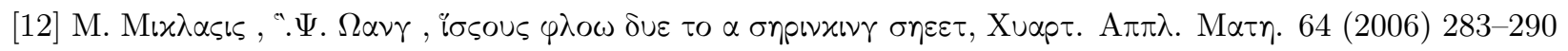

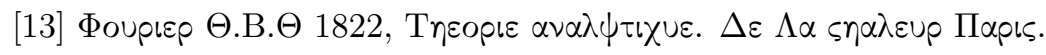

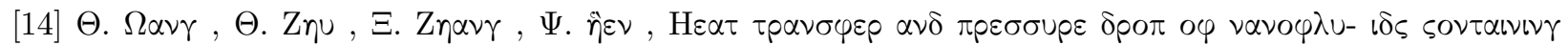

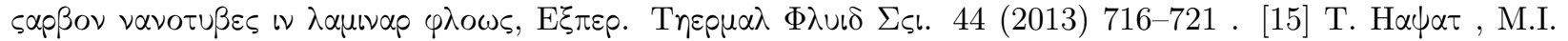

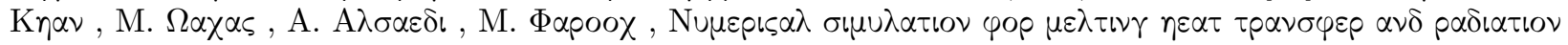

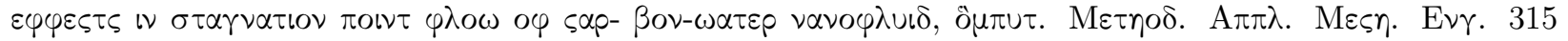
(2017) 1011-1024.

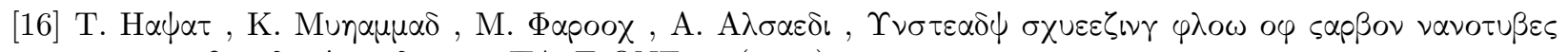

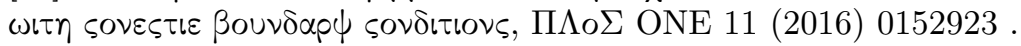

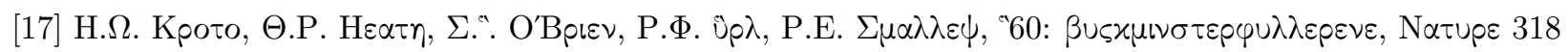
(1985) 162-163.

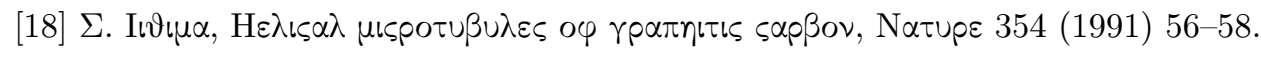

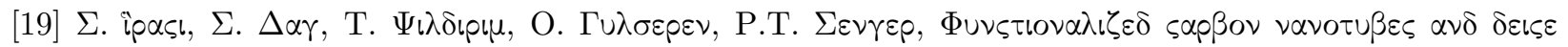

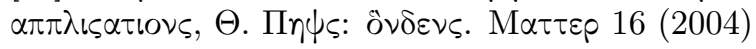

P901-P960.

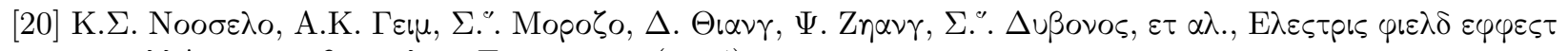

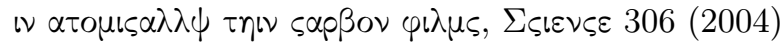

666-669.

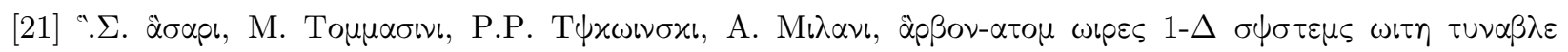

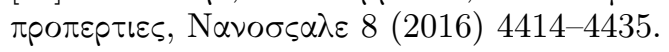

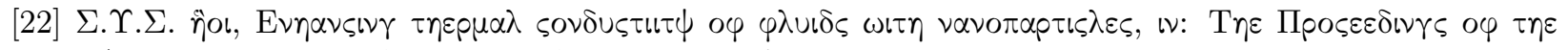

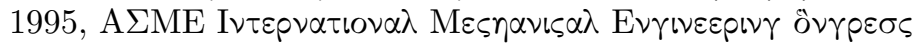

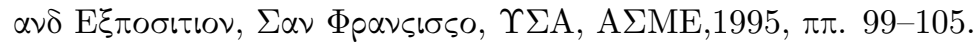

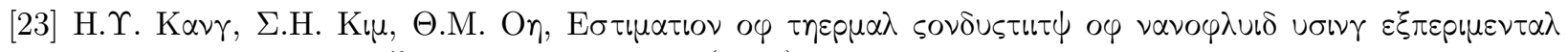

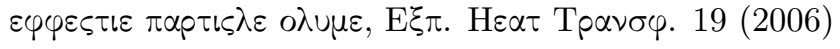

181-191.

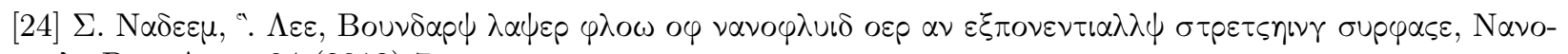

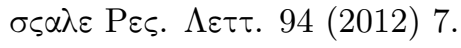

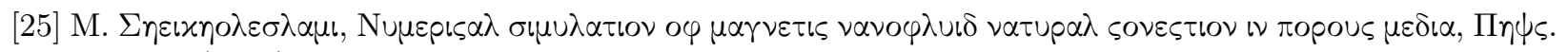

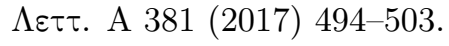

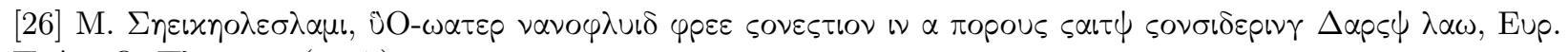

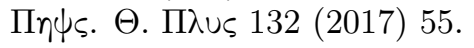

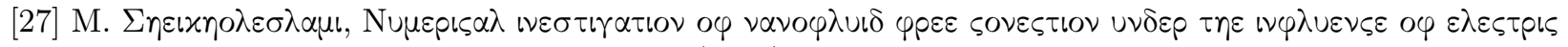

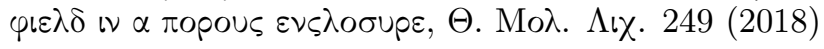

$1212-1221$.

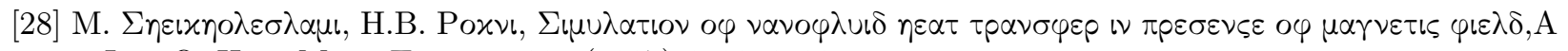

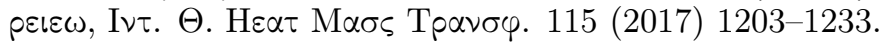




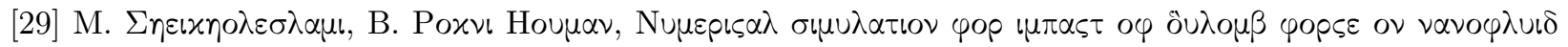

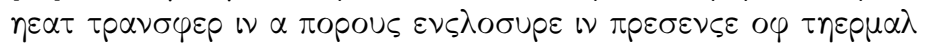

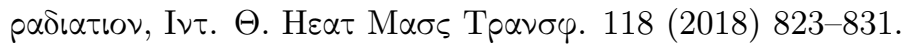

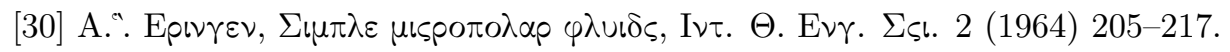

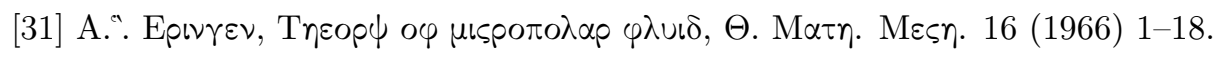

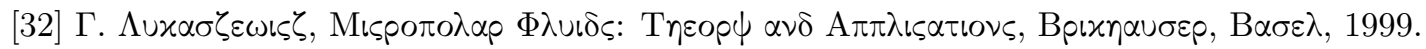

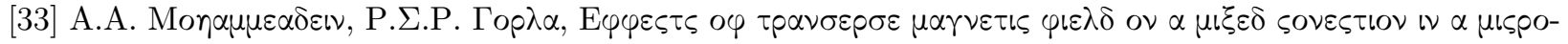
$\pi \circ \lambda \alpha \rho$

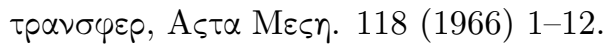

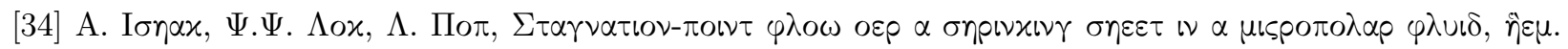

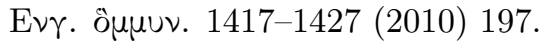

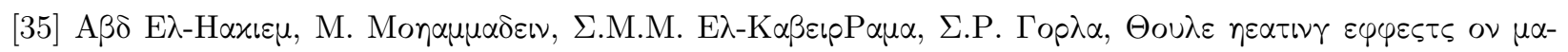

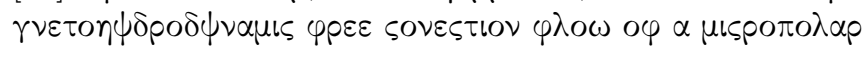

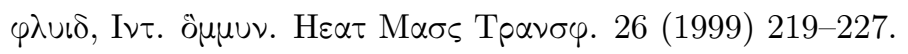

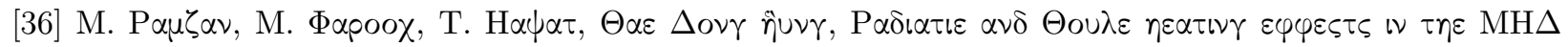

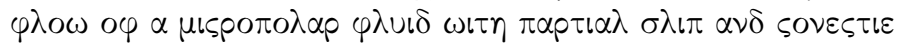

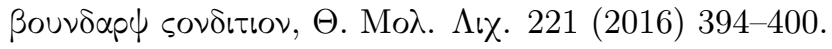

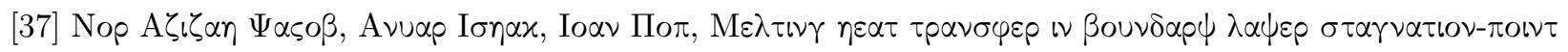

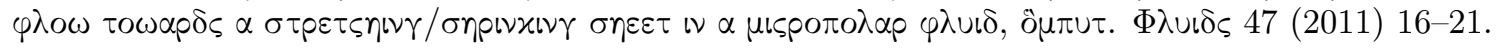

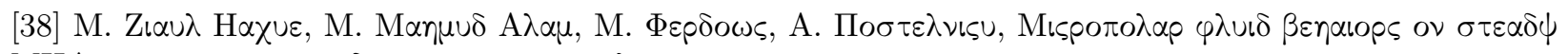

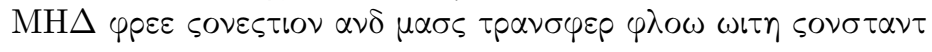

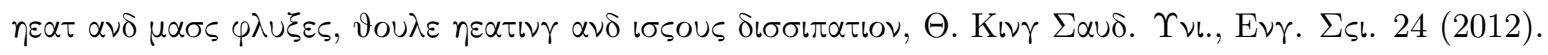

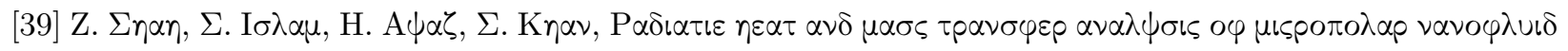

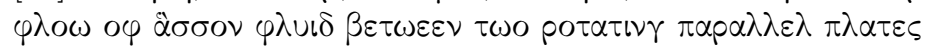

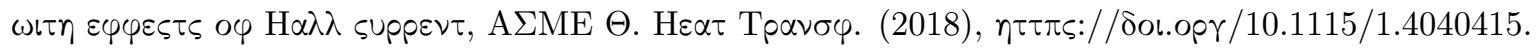

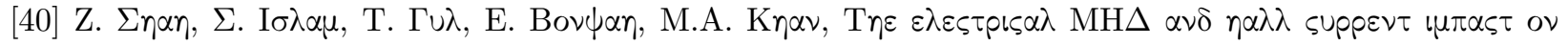

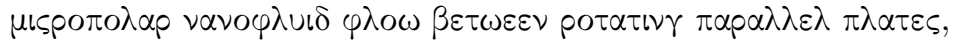

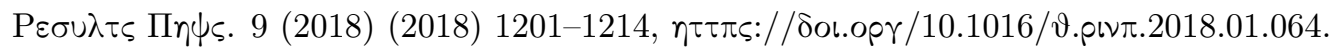

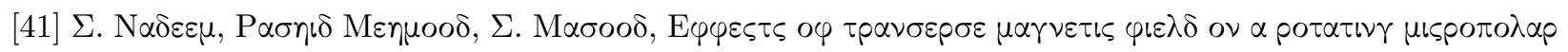

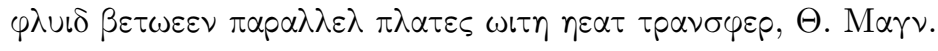

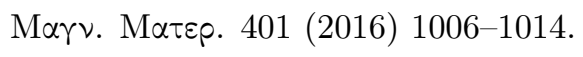

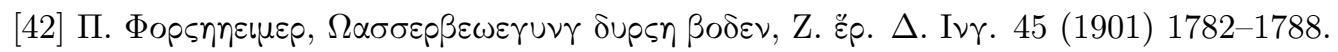

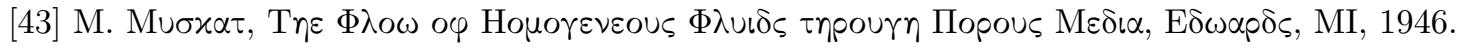

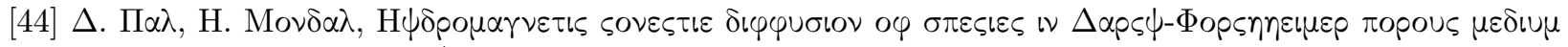

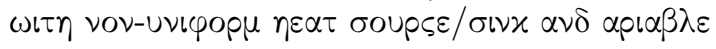

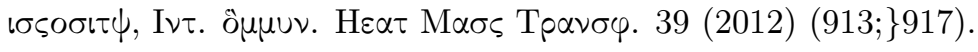

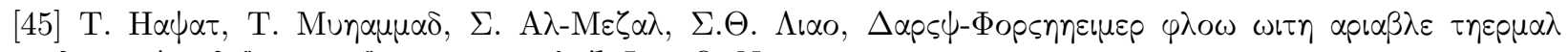

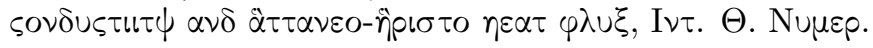




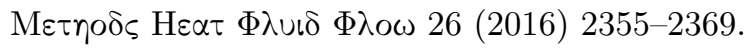

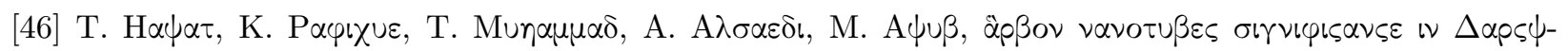

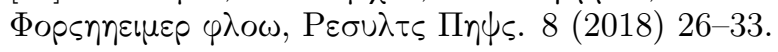

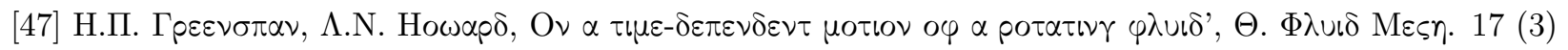
(1963) 385-404.

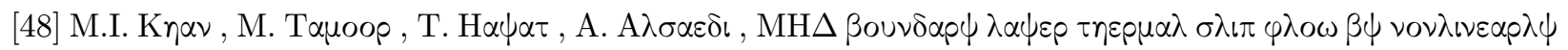

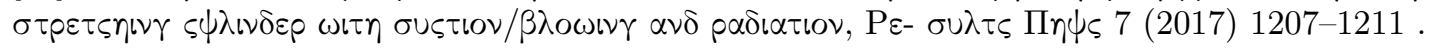

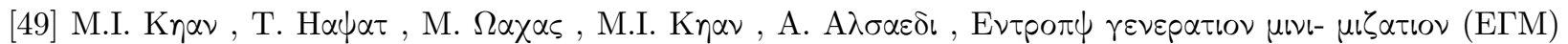

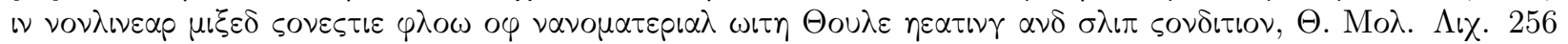
(2018) 108-120 .

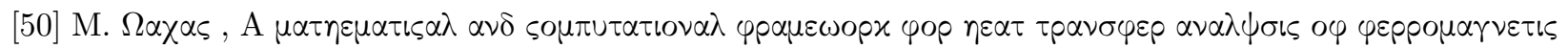

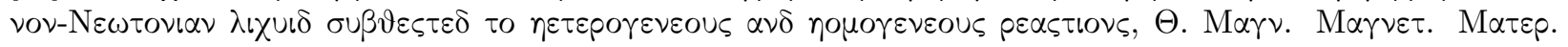
493 (2020) 165646 .

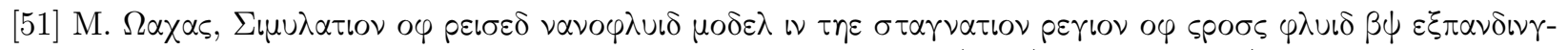

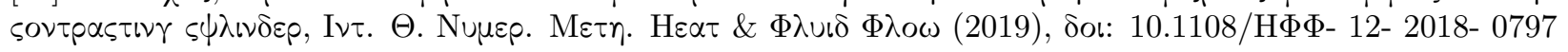

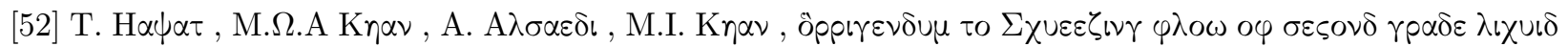

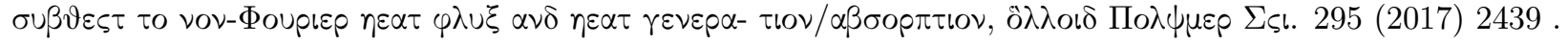

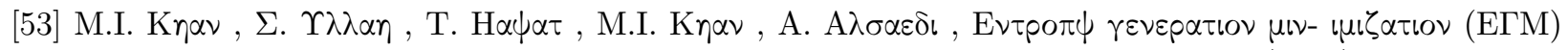

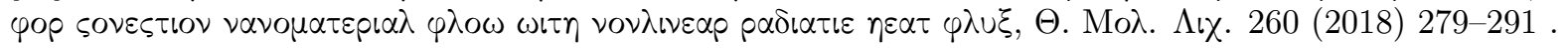

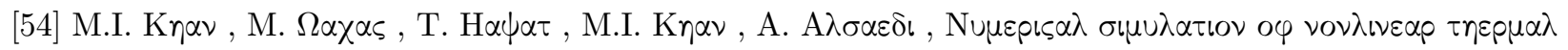

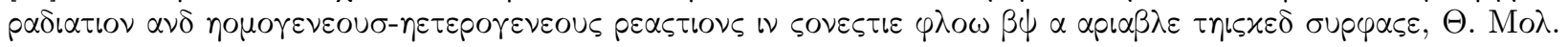
$\Lambda$ ix. 246 (2017) 259-267.

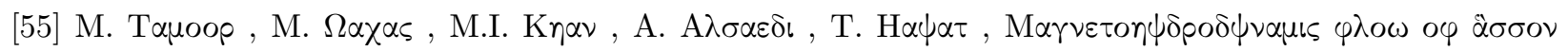

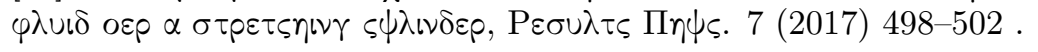

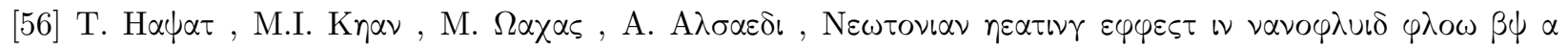

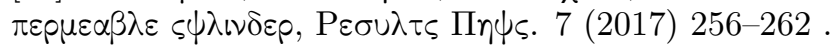

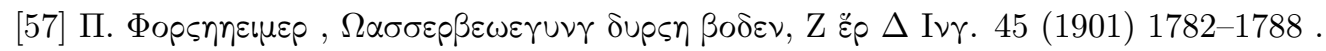

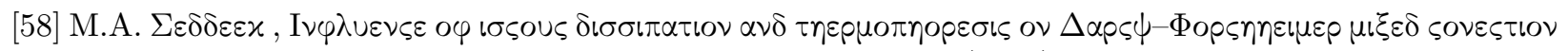

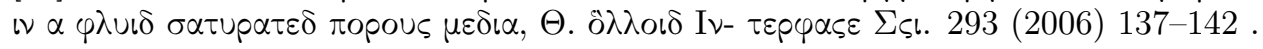

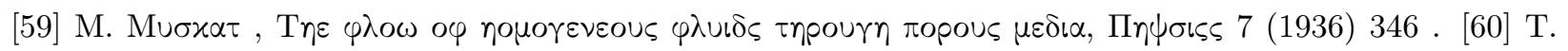

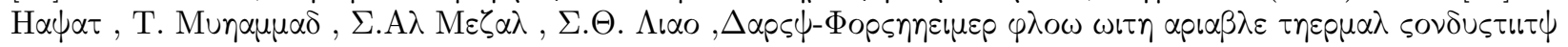

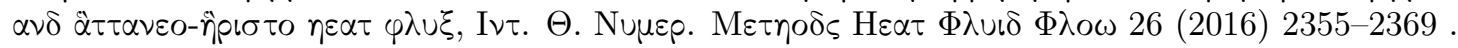

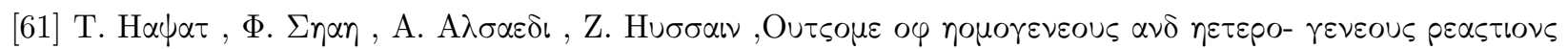
iv $\Delta \alpha \rho \varsigma \psi-\Phi о \rho \varsigma \eta \eta \varepsilon l \mu \varepsilon \rho ~ \varphi \lambda$ 2497-2505 .

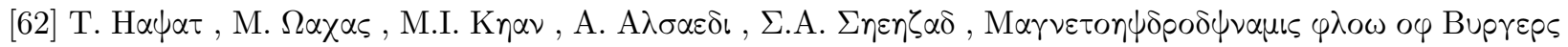

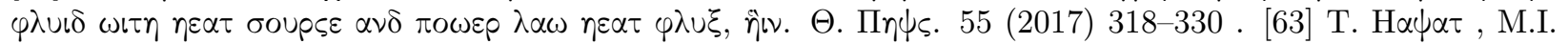

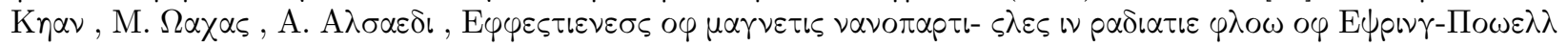

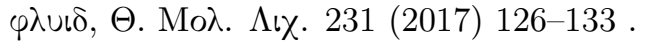

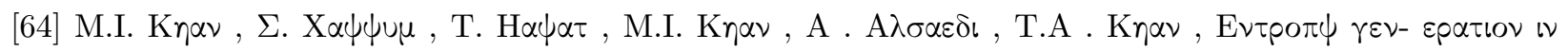

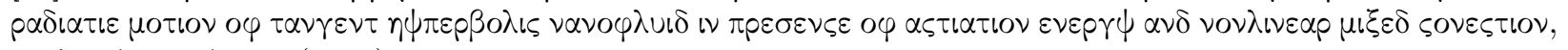

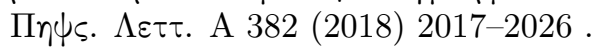




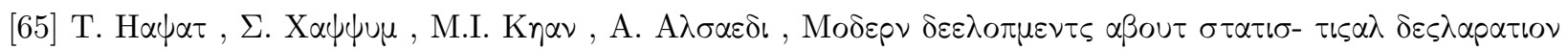

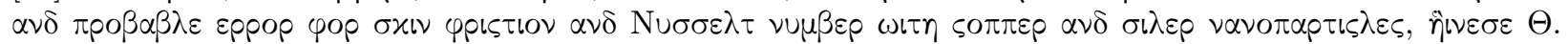
$\Pi \eta \psi$ s. 55 (2017) 2501-2513.

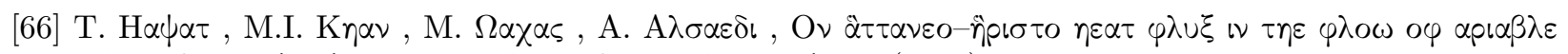

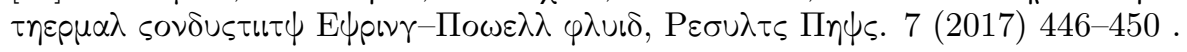

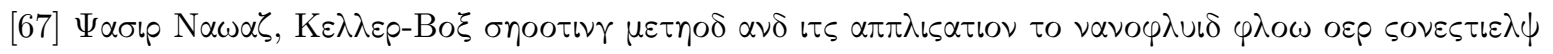

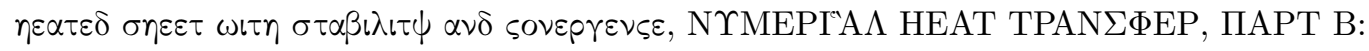

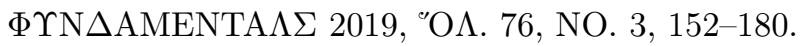

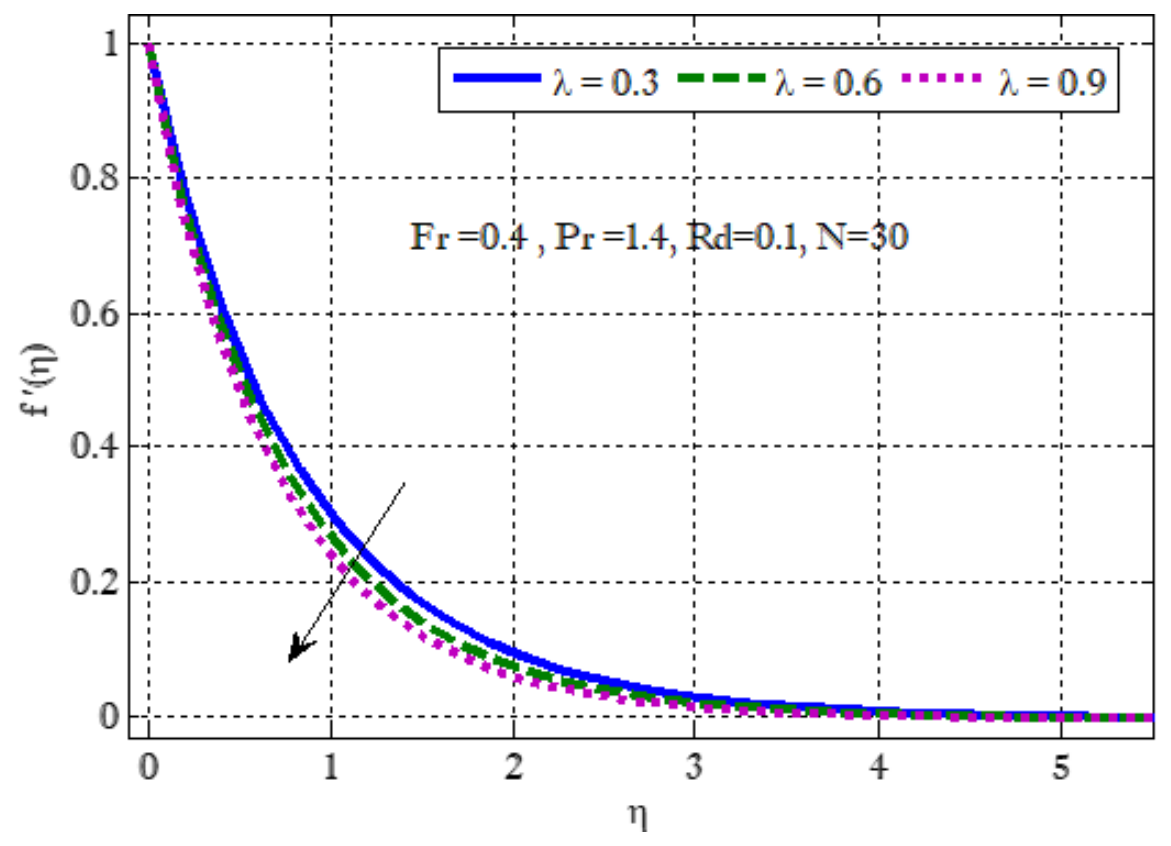



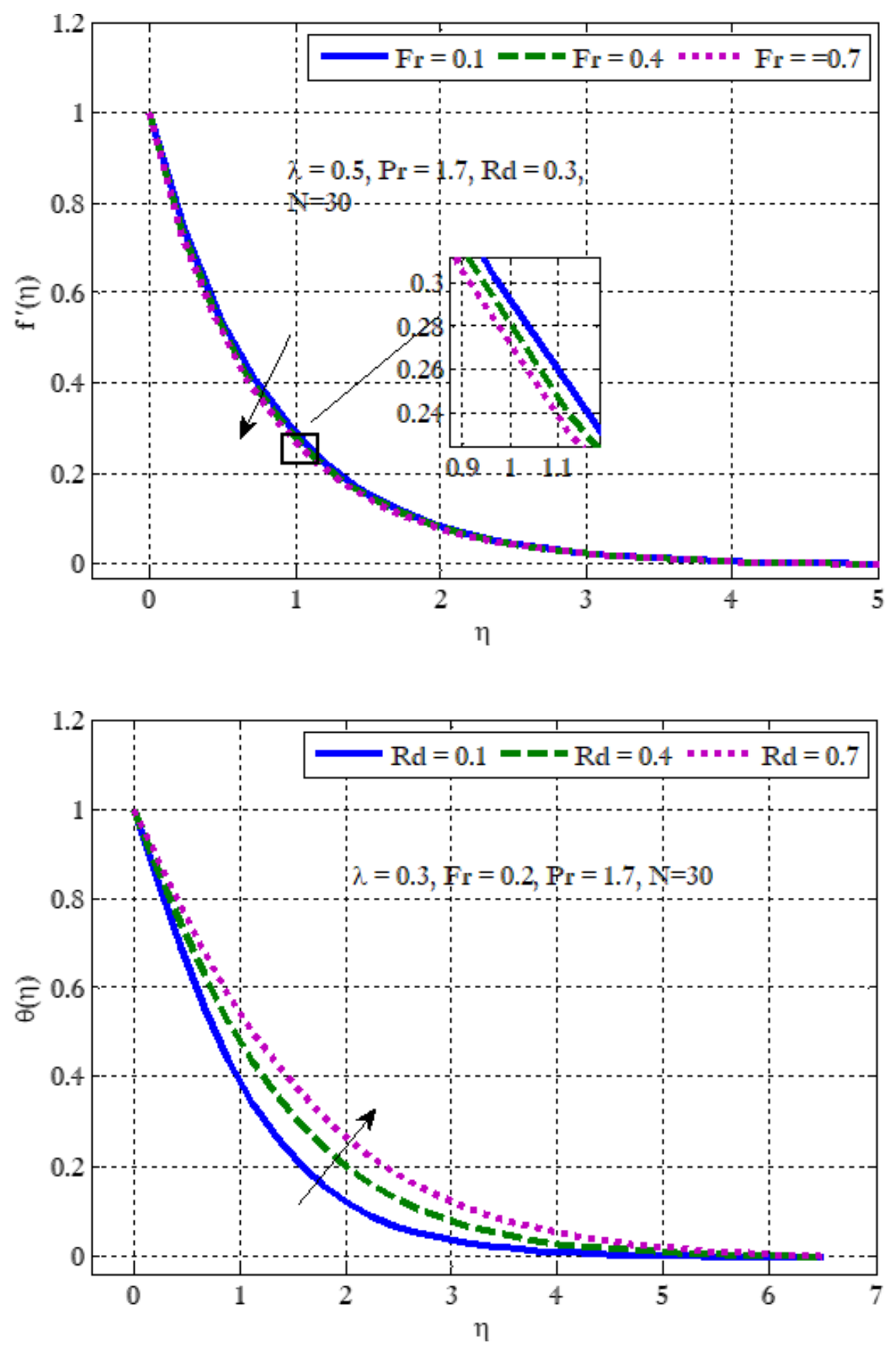

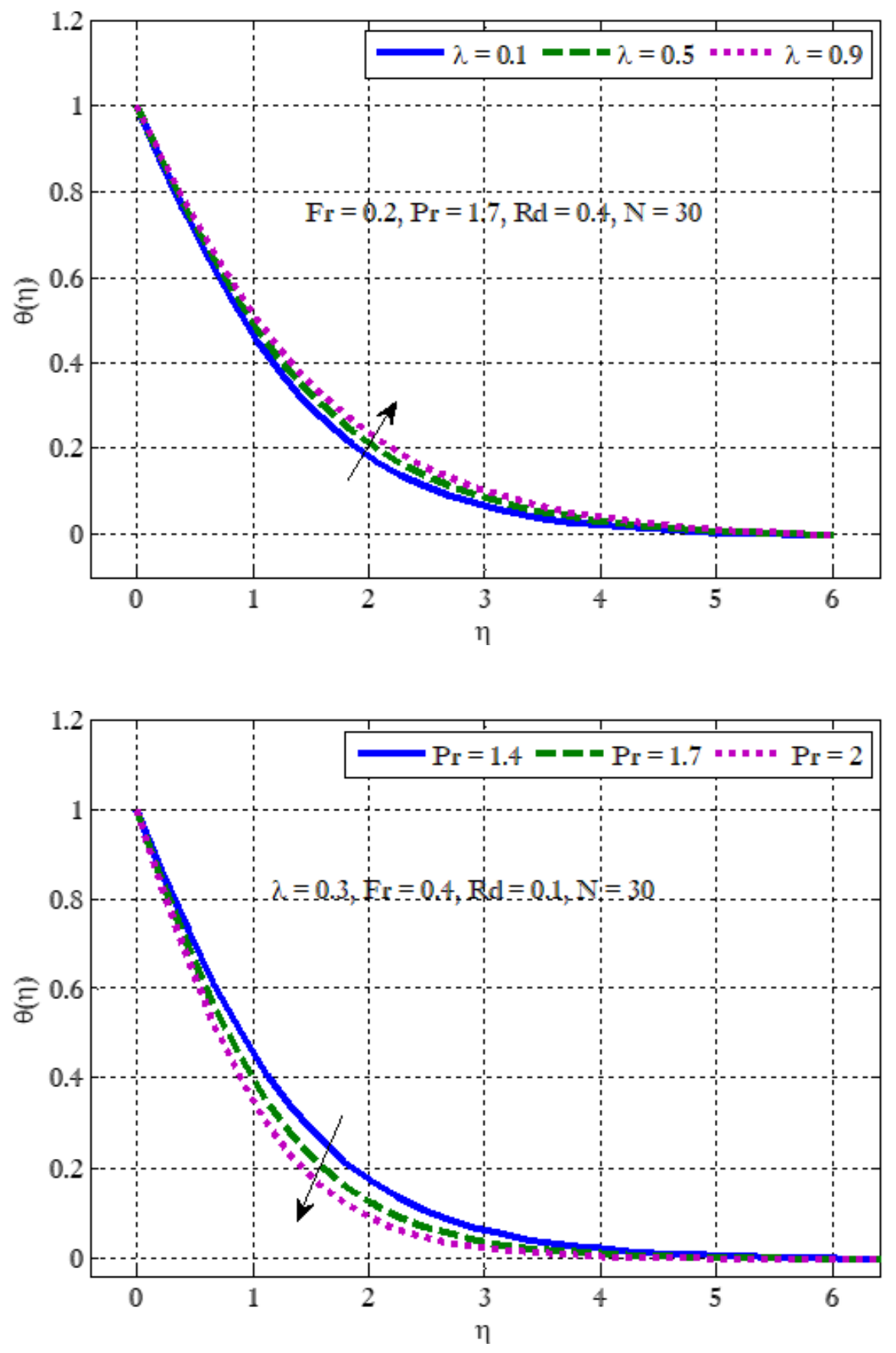

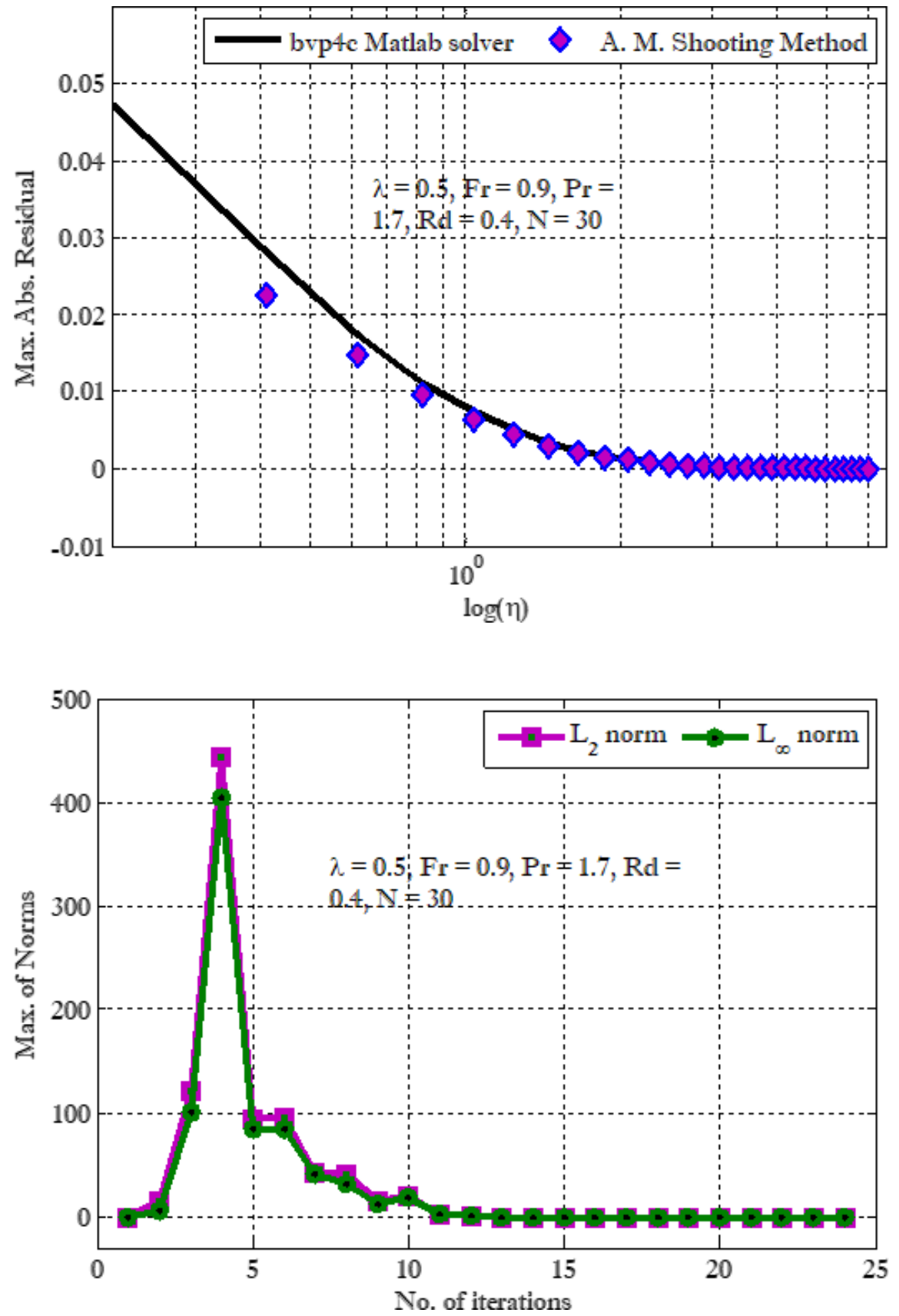


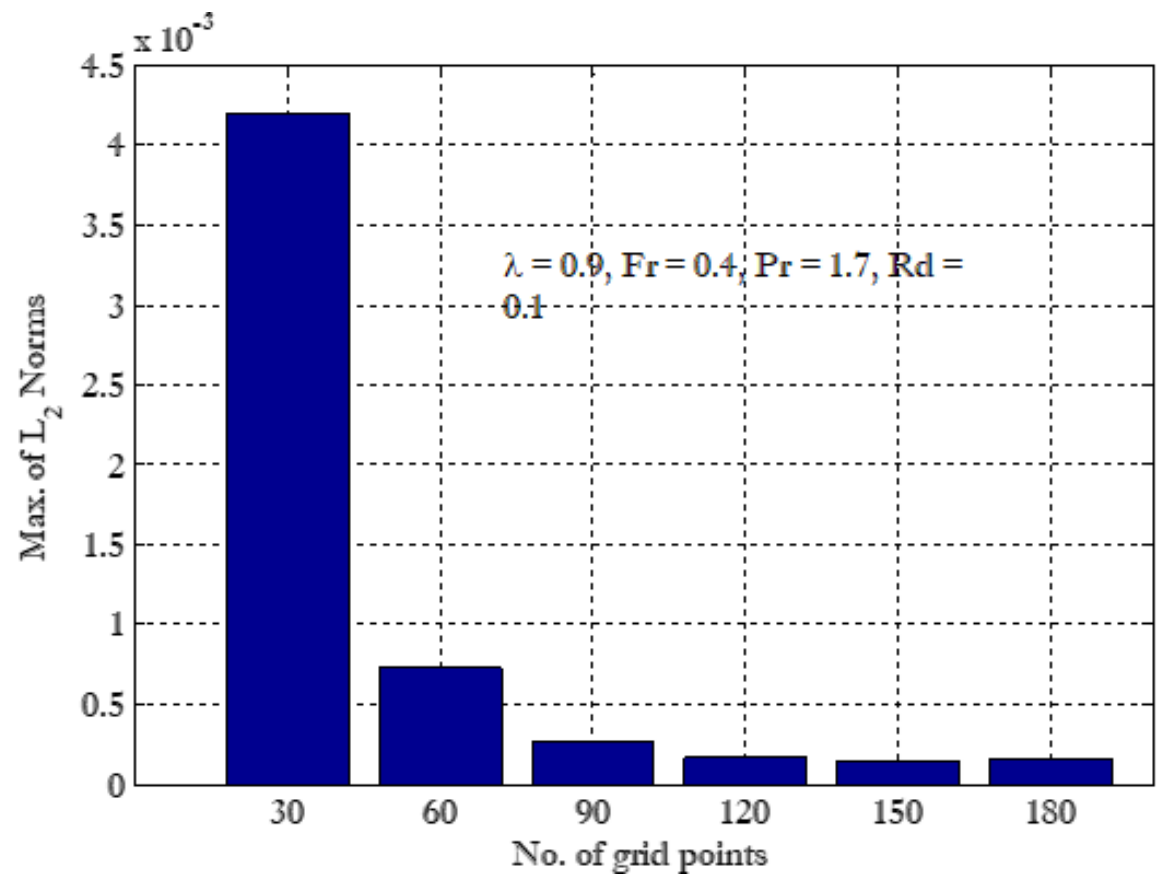



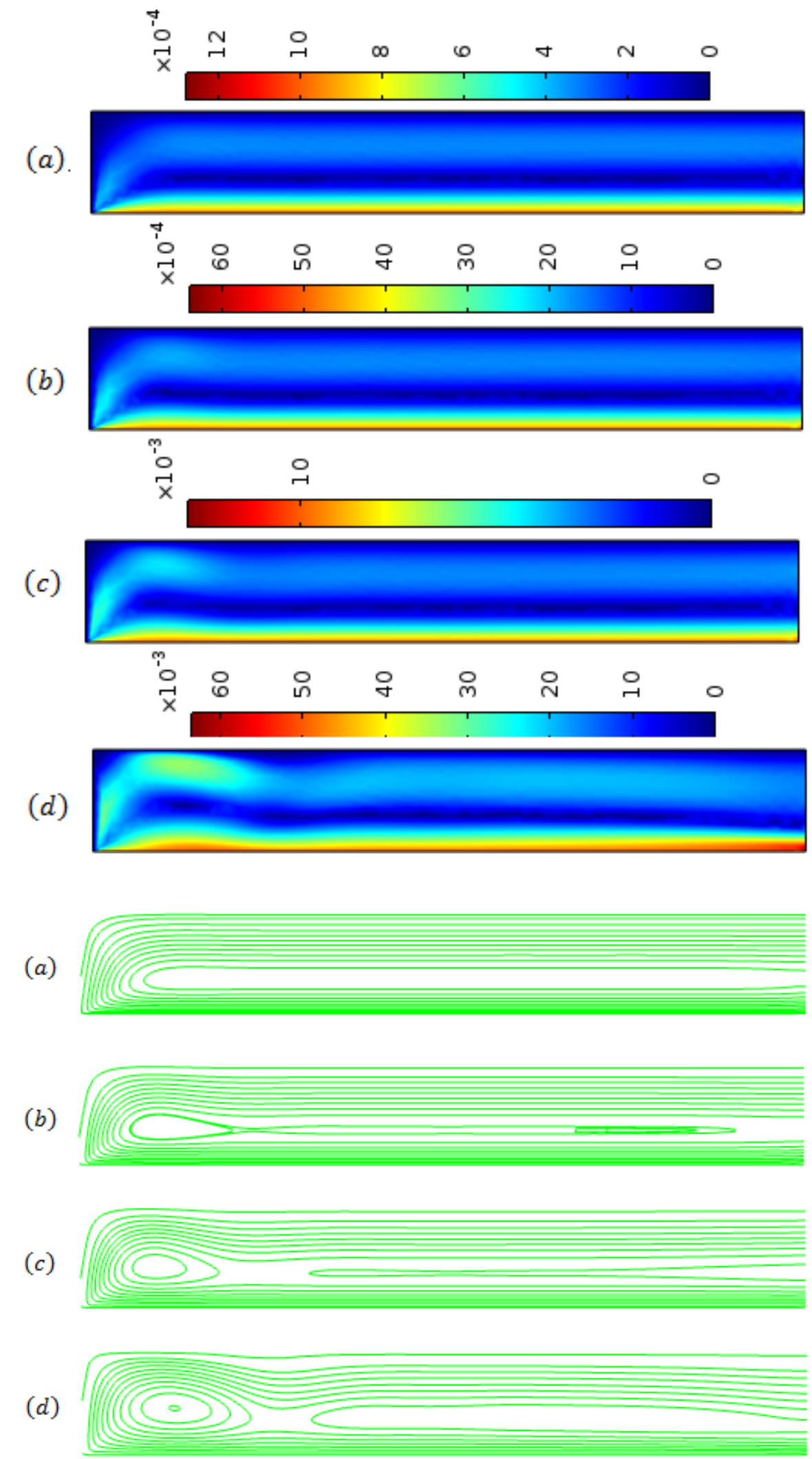

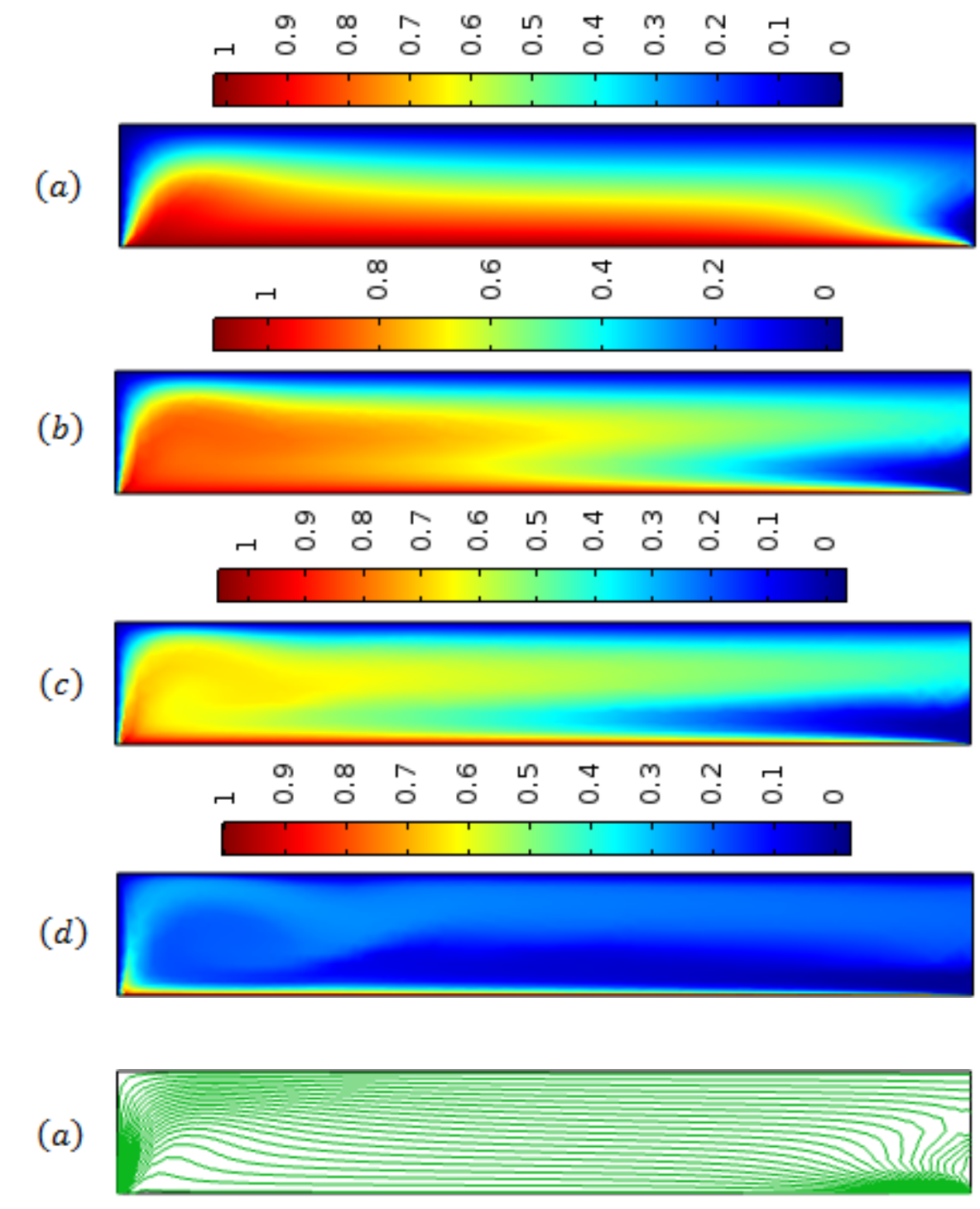

(b)

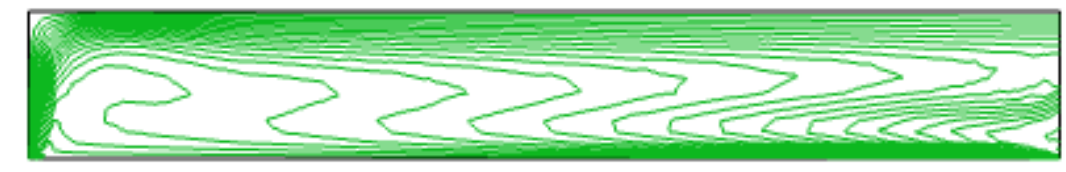

(c)
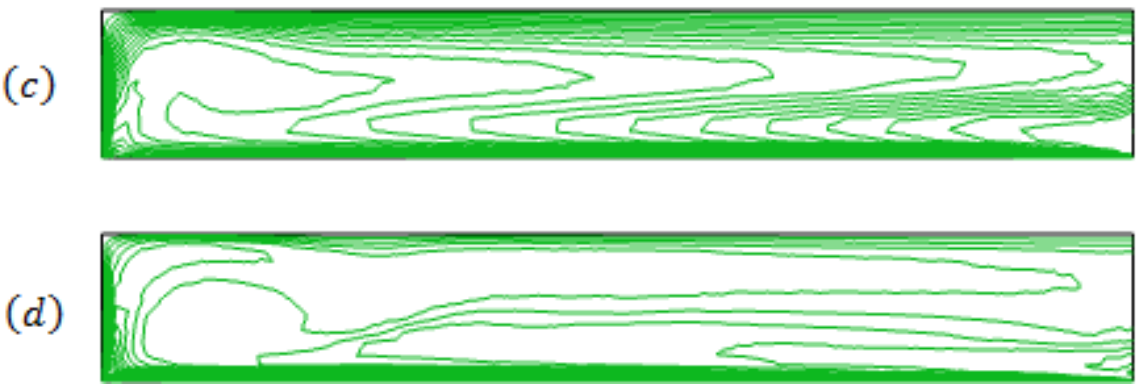